DRAFT VERSION JANUARY 24, 2012

Preprint typeset using $\mathrm{LT}_{\mathrm{E}} \mathrm{X}$ style emulateapj v. 5/2/11

\title{
GRAVITATIONAL-WAVE EMISSION FROM COMPACT GALACTIC BINARIES
}

\author{
Samaya Nissanke, ${ }^{1,2}$ Michele Vallisneri, ${ }^{1,2}$ Gijs Nelemans, ${ }^{3,4,5}$ Thomas A. Prince ${ }^{1,2}$ \\ Draft version January 24, 2012
}

\begin{abstract}
Compact Galactic binaries where at least one member is a white dwarf or neutron star constitute the majority of individually detectable sources for future low-frequency space-based gravitationalwave (GW) observatories; in addition, they form an unresolved continuum, the dominant Galactic foreground at frequencies below a few $\mathrm{mHz}$. A handful of ultra-compact binaries, observed at optical, ultraviolet and X-ray wavelengths, are known verification sources for space-based GW interferometers. Due to the paucity of electromagnetic observations, the majority of studies of Galactic-binary populations so far have been based on population-synthesis simulations. However, recent surveys have reported several new detections of compact binaries including double white dwarfs, providing new constraints for population estimates. In this article, we evaluate the impact of revised local densities of interacting white dwarf binaries on future low-frequency GW observations. Specifically: we consider five scenarios that explain these densities with different assumptions on the formation of interacting systems; we simulate corresponding populations of detached and interacting white dwarf binaries; we estimate the number of individually detectable GW sources and the magnitude of the confusion-noise foreground, in the case of two representative space-based detectors (LISA-like interferometers with armlengths of 1 and $5 \mathrm{Mkm}$ ). We confirm earlier estimates of thousands of detached-binary detections, but project only a few ten to a few hundred detections of interacting systems. We also confirm earlier estimates for the confusion-noise foreground (except in one scenario that explains smaller local densities of interacting systems with smaller numbers of progenitor detached systems). Last, we provide a general scaling argument that shows that the magnitude of the GW foreground can be derived robustly from the merger rate of Galactic white dwarf binaries, and depends only weakly on the structure of the Galaxy.

Subject headings: gravitational waves, Galactic binaries, AM CVn, data analysis, LISA
\end{abstract}

\section{INTRODUCTION}

Compact Galactic binaries with periods shorter than approximately five hours are of considerable interest as gravitational wave (GW) sources (Nelemans 2009; Marsh 2011). Such binaries include detached double white dwarf (DDWD) systems, short-period cataclysmic variables (CVs), and AM CVn systems (so named after their prototype, AM Canum Venaticorum; see Solheim 2010). These last are short-period binaries in which a white dwarf (WD) is accreting He-rich material from an $\mathrm{H}$-deficient companion. AM CVn systems are defined spectroscopically by the presence of strong absorption or emission He lines and the absence of $\mathrm{H}$ lines, which instead characterize "standard" CVs. In this paper we focus on those binaries that are evolving toward, or have evolved from, extremely short orbital periods, on the order of minutes. These include DDWD and AM CVn systems, sometimes referred to as "ultra-compact" binary systems. Such binaries have received considerable attention, among other reasons, as possible progenitors for Type Ia supernovae, R Coronae Borealis stars (Web-

${ }^{1}$ Jet Propulsion Laboratory, California Institute of Technologv, 4800 Oak Grove Drive. Pasadena. CA 91109

${ }^{2}$ Division of Physics, Mathematics, and Astronomy, California Institute of Technology, Pasadena, CA 91125

Department of Astrophysics, Radboud University Nijmegen Hevendaalseweg 135, NL-6525 AJ Nijmegen, the Netherlands

Institute for Astronomy, KU Leuven, Celestijnenlaan 200D, 3001 Leuven. Belgium

J Nikhef, Science Park 105, 1098 XG Amsterdam, 'The Netherlands bink 1984, Iben \& Tutukov 1984), and so-called ".Ia" explosions (Bildsten et al. 2007). Our understanding of their formation and evolution remains poor.

Together with ultra-compact X-ray binaries, DDWD and AM CVn systems will be the most numerous and individually brightest GW sources in the Galaxy for low-frequency $\left(10^{-5}-1 \mathrm{~Hz}\right)$ space-based GW detectors. Within this frequency band, observations suggest $5-25 \times$ $10^{6}$ DDWD systems and theoretical estimates predict $10^{5}-10^{7}$ AM CVn systems in our Galaxy (Maxted \& Marsh 1999, Nelemans et al. 2001a c, 2004).

The aim of this paper is to characterize the detectable GW emission from compact binaries in light of recent population estimates based on new observations. The first estimates were based solely on population-synthesis simulations; see App. A for a detailed discussion and review of the literature. Recent observations of AM CVn systems have provided new information that constrains earlier estimates. For instance, the identification of AM CVn systems among candidates selected from the Sloan Digital Sky Survey (SDSS) spectroscopic catalog allowed Roelofs et al. (2007b) to estimate the density of AM CVn systems by modeling the completeness of the catalog. A follow-up spectroscopic survey of a color-selected subset of the SDSS photometric database seems to find even fewer AM CVn systems than expected (Roelofs, 2008; Rau, 2010), leading to local density estimates smaller by 10-20 than suggested by population-synthesis studies.

To date, $27 \mathrm{AM}$ CVn systems have been presented in the literature; the most recent detections have been 
achieved in synoptic surveys, including a short-period system from Kepler (Fontaine et al. 2011), and a medium-period, outbursting system from the Palomar Transient Factory (Levitan et al. 2011).

Similarly, surveys of low-mass WD candidates selected from SDSS has led to the discovery of several shortperiod DDWDs that could be progenitors of AM CVn systems (Badenes et al. 2009: Mullally et al. 2009; Kulkarni \& van Kerkwijk 2010; Marsh et al. 2010). For example, the ELM (Extremely Low Mass) survey (Brown et al. 2010, Kilic et al.2011a; Brown et al. 2012) searches for companions around extremely low mass WDs $(\sim$ $\left.0.2 M_{\odot}\right)$. The ELM survey has approximately quintupled (with 19 new systems) the known sample of DDWDs that will merge within a Hubble time (Kilic et al. 2011b; Brown et al. 2012), providing evidence for at least one plausible AM CVn formation channel (see Sec. 2.1).

In this paper, we consider five scenarios for AM CVn formation that span plausible populations, and we study their consequences for space-based GW detectors by modeling the detection and subtraction of sources in simulated data streams. We consider two representative detectors: the standard LISA developed as a joint NASA-ESA project until 2011 (Prince et al. 2009, Jennrich 2009), and a descoped configuration with shorter armlengths of $1 \mathrm{Mkm}$, which is similar, but not equivalent, to the NGO/eLISA design studied by ESA in 2011 (Amaro-Seoane et al. 2012).

We find, first, that the number of detectable AM CVn systems decreases from $\sim 10,000$, as reported in previous assessments of LISA science, to $\sim 100-400$ (for one year of observation with a single interferometer - see App. B. Precise numbers depend on the assumptions used to explain how a postulated number of DDWD progenitors can result in the number of AM CVn systems seen in surveys. For the shorter detector, these numbers are reduced by a factor of a few. Second, we confirm that mostly DDWD systems determine the residual confusion foreground left after GW signals from detectable binaries are subtracted. The foreground does not change significantly from previous estimates, except in one scenario in which we explain the fewer observed AM CVn systems by reducing the space density of DDWD progenitors.

The rest of this paper is structured as follows. In Sec. 2 we discuss the current understanding of AM CVn formation. In Sec. 3 we outline the observations of AM CVn and DDWD systems in the Galaxy, and the resulting calibration of local space densities. In Sec. 4 we present our analysis: we introduce our explanatory formation scenarios in Sec. 4.1. discuss the space-based observation of GWs from Galactic binaries in Sec. 4.2, describe our simulations of GW source detection and subtraction in Sec. 4.3 and detail our results in Sec. 5. In Sec. 66we recapitulate our conclusions and propose possible future work. In the appendices we provide more detailed discussions of specific topics: in App. A we summarize previous analytical and numerical studies of the Galactic foreground for LISA, and give a general semi-analytical derivation of its magnitude and shape; in App. B we discuss GW detection and define our reference detector configurations; in App. Clwe provide more details about the fits of the GW confusion foreground obtained from our simulations. In the rest of the paper, we use the standard notation where $G$ is the gravitational constant and $c$ is the speed of light.

\section{THE FORMATION AND EVOLUTION OF COMPACT BINARY SYSTEMS IN THE GALAXY}

In the standard picture of binary-star evolution, compact binaries form following two consecutive masstransfer episodes; the loss of sufficient angular momentum by friction in the second common-envelope phase ensures sufficiently tight orbits for GW emission to influence dynamics. Here we concentrate on the processes leading to mass transfer and to the formation of $\mathrm{AM} \mathrm{CVn}$ systems.

The subsequent orbital evolution of compact binaries is driven by gravitational radiation, by mass transfer, by a dissipative coupling that can feed the angular momentum added through accretion back into the orbit, and by other poorly modeled processes such as wind loss, etc. Therefore, the rate of change $\dot{J}_{\text {orb }}$ of the orbital angular momentum comprises three or more competing components,

$$
\dot{J}_{\mathrm{orb}}=\dot{J}_{\mathrm{GR}}+\dot{J}_{\dot{\mathrm{M}}}+\dot{J}_{\tau_{s}} .
$$

Here $\dot{J}_{\mathrm{GR}}$ represents the loss of angular momentum to gravitational radiation,

$$
\dot{J}_{\mathrm{GR}}=-\frac{32}{5} \frac{G^{3}}{c^{5}} \frac{M_{1} M_{2} M}{a^{4}} J_{\mathrm{orb}}
$$

(for circular orbits) where $M_{1}$ and $M_{2}$ are the two binary masses $\left(M_{2}\right.$ is the donor's mass for mass-transferring systems), $a$ their separation, and $M=M_{1}+M_{2}$. Next, $\dot{J}_{\dot{\mathrm{M}}}=\sqrt{G M_{1} R_{h}} \dot{M}_{2}$, where $\dot{M}_{2}$ indicates the donor's mass-loss rate due to Roche-lobe overflow and $R_{h}$ defines the orbital radius around the accretor with the same specific angular momentum as the transferred mass (Verbunt \& Rappaport 1988).

Mass transfer can proceed either via an accretion disk or via "direct impact" (Marsh \& Steeghs 2002, Ramsay et al. 2002, Marsh et al. 2004). "Direct impact" occurs when the donor's mass stream hits the accreting star directly. The particular route depends on the initial separation in the binary following the ejection of the second envelope, and on the donor's entropy and equation of state. Typically, mass-transferring binaries with initial orbital periods greater than ten minutes form an accretion disk. As discussed below, the presence or absence of an accretion disk is critical to the stability of the system. Whether a disk is present or not, $\dot{J}_{\tau_{s}}$ denotes the change in the torque due to dissipative coupling, tidal or magnetic, between the accretor's spin and the orbital angular momentum during the accretor's synchronization timescale $\tau_{s}$ (we assume that the donor and orbit's spins are synchronized). In this work, we do not consider the change in angular momentum driven by other physical processes, such as losses due to winds or magnetic braking, because of current limitations in our understanding and modeling of such phenomena.

As the term "detached" in their very name implies, DDWD dynamics are determined solely by gravitational radiation and tides (see e.g. Piro 2011). By contrast, the formation, stability, and outcome of AM CVn systems depend on all three effects in Eq. (1). Three proposed channels exist for the formation of A $\mathrm{M}$ CVn systems, and each can vary in its efficiency. We describe them below. Following Nelemans et al. (2001a b, 2004), we assume that the first two channels listed below can operate with 
either "optimistic" or "pessimistic" efficiencies, resulting in different AM CVn numbers and period distributions.

\subsection{The DDWD channel}

A subclass of DDWDs with periods of several minutes becomes semi-detached when angular-momentum loss due to gravitational radiation drives the two bodies sufficiently close that the lower-mass WD fills its Roche lobe and mass transfer begins, either via direct impact or via an accretion disk. The stability of mass transfer depends critically on the mass ratio of the two initial WDs (requiring typically $q=M_{2} / M_{1} \lesssim 2 / 3$ ) and on whether spin-orbit tidal coupling feeds angular momentum accreted by the primary star back to the orbit; the strength of this coupling is characterized by the synchronization timescale $\tau_{s}$ (Marsh et al. 2004).

Under optimistic assumptions, strong tidal coupling stabilizes mass transfer, favoring the formation of stable AM CVn systems and resulting in fewer mergers. By contrast, under pessimistic assumptions there is no tidal coupling and many more DDWD systems merge. In the presence of a disk, the accreted material's angular momentum is mainly transferred back into the orbit by tidal forces at the outer edge of the disk. However, the disc's material at the inner edge still has sufficient angular momentum to spin up the accretor. Effects not considered here, such as magnetic braking (Farmer \& Roelofs 2010), may also impact the formation of AM CVn systems and favor the merger of the WDs.

If stable mass transfer proceeds, the donor WD loses mass and expands in size, while the system evolves to longer orbital periods. The minimum orbital period at which mass transfer can begin is $\sim$ two minutes for a 0.45 $M_{\odot}$ and $0.25 M_{\odot}$ binary. Maximum orbital periods for evolved systems in the Galaxy are estimated to be about 90 minutes; these correspond to very old systems whose orbits have slowly evolved over a Hubble time.

Surveys specifically targeted at low-mass WD [such as the ELM survey of WDs: Brown et al. (2010); Kilic et al. (2011a); Brown et al. (2011)] have resulted in the discovery of numerous DDWD systems with $q<2 / 3$, which are expected to get into contact within a Hubble time. Several of these newly discovered low-mass binaries will form stable mass-transferring AM CVn systems, even in the most pessimistic case of no tidal coupling (Brown et al. 2011).

\subsection{The Helium star-white dwarf channel}

In this channel, a WD accretes from an initially nondegenerate He star with mass $0.4-0.6 M_{\odot}$ before the $\mathrm{He}$ has been exhausted in the He-star core. He stars are produced from stars with masses greater than $2 M_{\odot}$ that lose their envelopes on the red-giant branch, and typically have lifetimes comparable to their main-sequence progenitors, allowing for mass transfer before evolution to the WD stage. Mass transfer is stable when the mass ratio of the donor He star to the accretor WD is less than approximately 1.2 (Tutukov \& Fedorova 1989, Ergma \& Fedorova 1990; see also Nelemans et al. 2001a). Due to mass loss, He burning in the donor's core quenches and at masses $\sim 0.2 M_{\odot}$ the star becomes semi-degenerate. The minimum orbital period reached by these systems is $\sim$ ten minutes.
It remains in question whether $0.1 M_{\odot}$ or $0.3 M_{\odot} \mathrm{He}$ accreted from a He star will destroy a CO WD by edgelit detonations (henceforth denoted ELDs; see Shen et al. 2010 and references therein). Nelemans et al. (2001a) distinguish between optimistic and pessimistic assumptions for this channel depending on whether double edge-lit detonations remove CO WD systems.

\subsection{The evolved Cataclysmic Variable channel}

In analogy to traditional $\mathrm{CVs}$, a so-called evolved $\mathrm{CV}$ comprises a main-sequence star which transfers mass to a WD or NS (Podsiadlowski et al. 2001). The difference is that mass transfer occurs as the donor star evolves off the hydrogen main sequence. However, it is thought that the evolved-CV channel contributes only marginally $(<2 \%)$ to the AM CVn population (Nelemans et al. 2004, Roelofs et al. 2007b) for systems with periods less than 1500s. 'Therefore, we do not consider this channel in this work, which focuses on the detectability of AM CVn systems by GW interferometers.

\section{OBSERVATIONS OF AM CVN AND DDWD SYSTEMS IN THE GALAXY}

We now turn our attention to recent population estimates of AM CVn systems based on observations. As mentioned, Roelofs et al. (2007b) estimate the total space density of AM CVn systems using observations of a source population identified by emission lines in SDSS. Characterizing selection effects for the six observed systems in SDSS is challenging. The orbital periods of these systems are generally shorter than predicted for the majority of AM CVn systems by population estimates, thereby requiring a significant extrapolation to estimate the space density of the entire population.

Table 1 in Roelofs et al. (2007b) presents the modeled and inferred AM CVn local space densities for different population-synthesis models. The inferred densities range from $1.2 \times 10^{-6}$ to $3.4 \times 10^{-6} \mathrm{pc}^{-3}$, assuming a thin-disk population with a scale height of $300 \mathrm{pc}$ for most systems. A comparison between the inferred and modeled densities immediately disfavors formation models such as the optimistic DDWD channel, and requires an overall reduction in the estimated number of Galactic AM CVn systems by a factor larger than ten. Roelofs et al. (2007b) hypothesize that lower AM CVn densities arise predominantly due to the lower efficiency of the major formation channels. Later in this paper we consider an alternative possibility, namely that systems observed by Roelofs et al. (2007b) may come from a distribution with scale height larger than conventional thin disks, with AM CVn progenitors born early in the history of the Galaxy, before the thin disk was fully developed.

Although the number of known DDWD systems that will merge within a Hubble time has increased to 24, an approximate factor of five, in the last few years (two eclipsing systems are now known; the ELM survey has uncovered the eclipsing DDWD binary with the shortest known period of 12.7 minutes), a detailed population analysis of such systems has yet to be undertaken. More recently, on the basis of the assumed Galactic-disk model, the ELM survey predicts $40 \mathrm{kpc}^{-3}$ of DDWDs where at least one WD has a mass $<0.25 M_{\odot}$, with a factor of two uncertainty in correction factors (Brown et al. 2011). This is much lower than population-synthesis results. On 
the other hand, those results show good agreement with observations made by the Supernova type Ia Progenitor (SPY) survey. The SPY radial-velocity survey estimated the local space density of WDs to be $4.8 \times 10^{-3} \mathrm{pc}^{-3}$ (Holberg et al. 2008), with $\sim 100$ new DDWDs found (with orbital periods less than ten days) from a sample of 1000.

\section{OUR ANALYSIS}

We now describe our analysis: in Sec. 4.1 we explain our choices for WD binary populations; in Sec. 4.2 we discuss GW emission from binaries; and in Sec. 4.3 we describe our simulations of binary detection and subtraction in detector data. Sec. 5 below presents the resulting estimates of the number of detectable systems and of the residual confusion-noise foreground.

\subsection{WD binary catalogs}

In our analysis we considered several different assumptions on AM CVn formation, generated simulated binary catalogs and detector datasets for each, and estimated the resulting binary detection and subtraction prospects. Among the large number of cases that we investigated, here for conciseness we report on five scenarios that we believe are representative of the range of possibilities given the constraints of current observations. Two of the scenarios represent upper or lower bounds on predictions from population-synthesis models (cases 1 and 2); two others attempt to reproduce SDSS-estimated AM CVn densities (cases 3 and 4 below); a fifth (case 5) explores a Galactic population with a larger scale height at times earlier than 7 Gyr ago. As discussed below, the modified-Galactic-disk scenario (case 5) should be consistent with SDSS observations; however, this agreement is difficult to quantify as the SDSS-estimated densities given in Roelofs et al. (2007b) are derived assuming a standard disk model.

We generate DDWD and AM CVn catalogs with the SeBa population-synthesis code (Portegies Zwart \& Verbunt 1996, Nelemans et al. 2001b, 2004; Toonen et al. 2011), with the following assumptions and parameters: i) the time- and position-dependent star formation rate (SFR) based on Boissier \& Prantzos (1999) (see Nelemans et al. 2004); ii) initial primary masses distributed with power-law index -2.5 (see Kroupa et al. 1993); iii) a flat initial mass-ratio distribution; iv) a flat distribution for the logarithm of the semimajor axis, up to a radius of $\left.10^{6} R_{\odot} ; \mathrm{v}\right)$ eccentricities distributed as $\left.P(e) \propto e^{2} ; \mathrm{vi}\right)$ a $50 \%$ binary fraction in the initial population of mainsequence stars. We expect a factor of two difference in population-synthesis results obtained using different assumptions and initial parameters than those stated above. Each system is described by seven parameters: the binary masses $M_{1}$ and $M_{2}$, the orbital period $P_{\text {orb }}$, the mass-transfer rate $\dot{M}$, the Galactic latitude $l$ and longitude $b$, and the luminosity distance $d$ to the Solar System.

Following Nelemans et al. (2004), we distribute single and binary stars according to a Galactic model that comprises both disk and bulge components. ${ }^{6}$ The density of

6 The bulge is not included in the analysis of Roelofs et al. (2007b). Furthermore, both Ruiter et al. (2009) and Yu \& Jeffery (2010) also include a halo component; however, the impact of halo DDWD systems seems negligible for GW interferometers. systems in the disk is

$$
\rho_{\text {disk }}(R, Z)=\rho_{\mathrm{BP}}(R) \operatorname{sech}^{2}\left(\frac{Z}{Z_{\text {disk }}}\right) \mathrm{pc}^{-3},
$$

where $R$ is the cylindrical radius from the Galactic center; $\rho_{\mathrm{BP}}(R)$ results from integrating the time-dependent plane-projected SFR of Boissier \& Prantzos (1999) [see Eqs. (1) and (2) of Nelemans et al. (2004)]; $Z$ is the perpendicular distance from the Galactic plane; and $Z_{\text {disk }}$ is the disk's scale height. The density of systems in the spherical bulge is given by

$$
\rho_{\text {bulge }}(r) \propto e^{-\left(r / r_{\text {bulge }}\right)^{2}} \mathrm{pc}^{-3},
$$

where $r$ is the spherical distance from the Galactic center; $r_{\text {bulge }}$ is the characteristic radius of the bulge; and Eq. (4) is normalized so that there are as many bulge systems as disk systems in the inner $3 \mathrm{kpc}$ of the Galaxy, with a current total mass of $2.6 \times 10^{6} M_{\odot}$.

For the first four scenarios we set $Z_{\text {disk }}=300 \mathrm{pc}$ and $r_{\text {bulge }}=0.5 \mathrm{kpc} ; \rho_{\mathrm{BP}}(R)$ is fit well by a density $\propto \exp \left(-R / R_{\text {disk }}\right)$ with $R_{\text {disk }} \approx 2.5 \mathrm{kpc}$ for DDWD systems and $R_{\text {disk }} \approx 2.2-2.4 \mathrm{kpc}$ for $\mathrm{AM} \mathrm{CVn}$ systems. In the last scenario we explore a modified model of the Galactic disk with a larger scale height for binaries that are produced at earlier times ( $>7$ Gyr ago).

For all but one scenarios we use the DDWD catalog analyzed in Nelemans et al. (2004), with local density $1.2 \times 10^{-5} \mathrm{pc}^{-3}$, and orbital periods as large as $5.5 \mathrm{hrs}$ $\left(f_{\mathrm{GW}}=10^{-4} \mathrm{~Hz}\right)$. Binaries at longer periods are not typically detectable by GW instruments because detector noise is higher at low frequencies; they are therefore not considered further in this analysis. All the catalogs of AM CVn systems, by contrast, show the effects of our different assumptions on AM CVn formation:

Case 1: upper bound (strong tidal coupling and inefficient ELD events). This catalog includes AM CVn systems that have formed via the DDWD channel operating with strong tidal coupling and the $\mathrm{He}$ star-WD channel with few ELD events (see Nelemans et al. 2001b, 2004). Although the resulting AM CVn local density is higher $\left(1.9 \times 10^{-5} \mathrm{pc}^{-3}\right)$ than SDSS-estimated values, past investigations of Galactic WD binaries as LISA sources have all used variants of this scenario (see, e.g., Edlund et al. 2005. Timpano et al. 2006). Thus, this case provides a meaningful comparison between our study and previously published results, which may vary depending on their idealizations of "detection," "resolution," etc. (see Sec. 4.3).

Case 2: lower bound (weak tidal coupling and efficient ELD events). This catalog includes AM CVn systems that have formed via the DDWD channel operating with minimal tidal coupling and the $\mathrm{He}$ star-WD channel with many ELD events (see Nelemans et al. 2001b, 2004). The AM CVn local density is $6.0 \times 10^{-6} \mathrm{pc}^{-3}$, a factor of two greater than SDSS-estimated values (Roelofs et al. 2007a), and fall within the factor of two uncertainty of population-synthesis results.

Case 3: mild tidal coupling and no ELD events. This catalog includes AM CVn systems that have 
formed via only the DDWD channel, where mild tidal coupling $\left(\tau_{s}=2\right.$ years) between the WDs allows for stable mass transfer in up to $10 \%$ of systems compared to the optimistic channel of Case 1. We assume that the He star-WD channel is suppressed. [However, observations discussed in (Roelofs et al. 2007a) suggest the presence of hot donors, which imply either that the He starWD channel is active with core He burning having been quenched only recently, or that following the second common-envelope ejection, the initial DDWD orbital separation is so small that the donor WD cannot cool within the gravitationalradiation timescale.] The AM CVn local density is $2.5 \times 10^{-6} \mathrm{pc}^{-3}$.

Case 4: fewer progenitor DDWDs. In this scenario we explain the SDSS-estimated AM CVn densities by postulating a $1 / 5$ reduction in the number of their DDWD progenitors. This reduction in DDWD systems is consistent with existing observations and with the factor of two uncertainty introduced in population-synthesis models by our limited understanding of binary evolution and Galactic structure. This catalog also includes AM CVn systems that have formed both via the DDWD channel with mild tidal coupling $\left(\tau_{1}=1\right.$ year $)$ and via the pessimistic He star-WD channel (see Nelemans et al. 2001b, 2004); their densities are also reduced by $1 / 5$ (across all periods). The DDWD and AM CVn local densities are $2.4 \times 10^{-6}$ and $1.2 \times 10^{-6} \mathrm{pc}^{-3}$.

Case 5: modified Galactic-disk model. In this scenario we place older systems in a thicker disk. With the caveat of small-number statistics, AM CVn systems identified by emission lines in SDSS have inferred projected velocities $>70 \mathrm{~km} \mathrm{~s}^{-1}$, hinting that AM CVn systems with longer periods may originate from a disk with a thick component, or at least from a thin disk with a larger scale height. Indeed, such a distribution of AM CVns at birth is consistent with our current (if everchanging) understanding of the spatial and temporal evolution of thin and thick galaxy disks (Scannapieco et al. 2011; Jurić et al. 2008; Siebert et al. 2011; Bovy et al. 2011). Observational constraints are unfortunately limited (see Napiwotzki 2009 for a discussion on the thin-disk, thick-disk, and halo populations of single WDs). As an illustrative example, we altered the disk component of Eq. 3 to have $Z_{\text {disk }}=1250 \mathrm{pc}$ for systems older than $7 \mathrm{Gyr}$, $Z_{\text {disk }}=300 \mathrm{pc}$ for systems younger than $7 \mathrm{Gyr}$, and $r_{\text {bulge }}=0.5 \mathrm{kpc}$ as for cases $1-4$. This effectively places all systems older than 7 Gyr in a thick disk, which includes the great majority of longer-period systems. In addition, we assumed that AM CVn systems only formed via the DDWD channel. The DDWD and AM CVn local densities are $1.2 \times 10^{-5}$ and $3.8 \times 10^{-6} \mathrm{pc}^{-3}$.

For each scenario, Table 1 shows the numbers of AM CVn and DDWD systems in the catalogs, as well as their local density $\rho_{0}$ (i.e., the Galactic mid-plane density at $8.5 \mathrm{kpc}$ from the Galactic center).

\subsection{Detecting gravitational waves from WD binaries}

Compact Galactic binaries in the low-frequency band emit quasi-monochromatic GWs, with frequency drifts of (at most) several frequency bins for year-long observation. In this paper, we model compact-binary waveforms by including only the dominant quadrupolar emission (Peters \& Mathews 1963), with instantaneous strain amplitude

$$
h=10^{-21}\left(\frac{\mathcal{M}_{z}}{M_{\odot}}\right)^{5 / 3}\left(\frac{P_{\text {orb }}}{\mathrm{hr}}\right)^{-2 / 3}\left(\frac{d}{\mathrm{kpc}}\right)^{-1},
$$

where $\mathcal{M}_{z}=(1+z) \mu^{3 / 5} M^{2 / 5}=(1+z) \mathcal{M}$ is the redshifted chirp mass (with $M$ the total mass, $\mu$ the reduced mass, $\mathcal{M}$ the chirp mass, and $z$ the redshift), $d$ is the luminosity distance to the source, and $P_{\text {orb }}$ is the orbital period of the binary.

Figure 1 shows the number density as a function of $h$ [the GW strain of Eq. (5)], and $f=2 / P_{\text {orb }}$ [the GW frequency], for the different DDWD and AM CVn catalogs of each of our scenarios. The colored dots indicate detected binaries (see Sec. 4.3): green shows DDWDs, red shows AM CVn systems (from the DDWD channel) and blue shows AM CVn systems (from the He-star-WD channel).

Binaries with orbital periods shorter than approximately 20 minutes will appear as isolated signals in yearlong datasets of space-based GW observatories, and will be detectable when the signals are sufficiently strong compared to instrument noise at the same frequency. By contrast, most binaries with longer orbital periods will not be detected individually, because they will be too dense in frequency space to tell apart. These will produce a noise-like confusion foreground that will affect the detection and parameter estimation of extra-Galactic GW sources such as massive-black-hole binaries. Nevertheless, the brightest binaries will be detected above the confusion foreground.

Looking at Fig. 1, we see that DDWD systems have larger densities than AM CVn systems, and larger GW strains (because they have greater chirp masses). Thus it is DDWD systems that are principally responsible for the shape and strength of the GW foreground from WD binaries (see Sec. 5.2). We note also that all AM CVn populations show an overall low-frequency cutoff at $\log _{10} f=-3.5$, due to the age of the Universe.

A simplified (but roughly correct) description of GW data analysis is that GW signals will be detectable when their signal-to-noise ratio, SNR, is greater than a detection threshold $\mathrm{SNR}_{\text {thr }}$ chosen to yield a minimal number of false alarms. SNR is the ratio of signal strength to rms instrument noise (plus confusion noise, if present) at the same frequency. For LISA-like interferometric detectors, the yearly orbit and rotation of the spacecraft constellation imprint the signals with frequency and amplitude modulations that encode the sky position of the source. Detector response is further complicated in frequencydependent fashion by Time-Delay Interferometry (TDI), the technique used to subtract the otherwise overwhelming laser frequency noise (thus, SNR would be evaluated for the TDI signal with respect to TDI noise). See App. B for an overview of LISA-like detectors, their response to GWs, and the theory of probabilistic signal detection 
TABLE 1

TOTAL NUMBER AND LOCAL DENSITY OF COMPACT-BINARY SYSTEMS OF DIFFERENT CLASSES (DETACHED, AM CVN FROM THE DDWD CHANNEl, AM CVN From the He-STAR-WD CHANNEL) in OUR Simulated CATAlOGS FOR CASES 1-5.

\begin{tabular}{crrr}
\hline \hline & \multicolumn{3}{c}{ number in catalog / local space density [pc ${ }^{-3}$ ] } \\
& DDWD & AM CVn (DDWD ch.) & AM CVn (He-star-WD ch.) \\
\hline case 1 & $26,084,411 / 1.2 \times 10^{-5}$ & $23,025,764 / 1.9 \times 10^{-5}$ & $11,197,735 / 9.0 \times 10^{-6}$ \\
case 2 & $26,084,411 / 1.2 \times 10^{-5}$ & $261,840 / 2.1 \times 10^{-7}$ & $6,643,091 / 5.9 \times 10^{-6}$ \\
case 3 & $26,084,411 / 1.2 \times 10^{-5}$ & $3,178,553 / 1.1 \times 10^{-6}$ & none $/ 0$ \\
case 4 & $5,217,866 / 2.4 \times 10^{-6}$ & $994,194 / 2.6 \times 10^{-7}$ & $1,328,909 / 1.2 \times 10^{-6}$ \\
case 5 & $26,084,509 / 1.2 \times 10^{-5}$ & $23,025,764 / 3.8 \times 10^{-6}$ & none $/ 0$ \\
\hline \hline
\end{tabular}
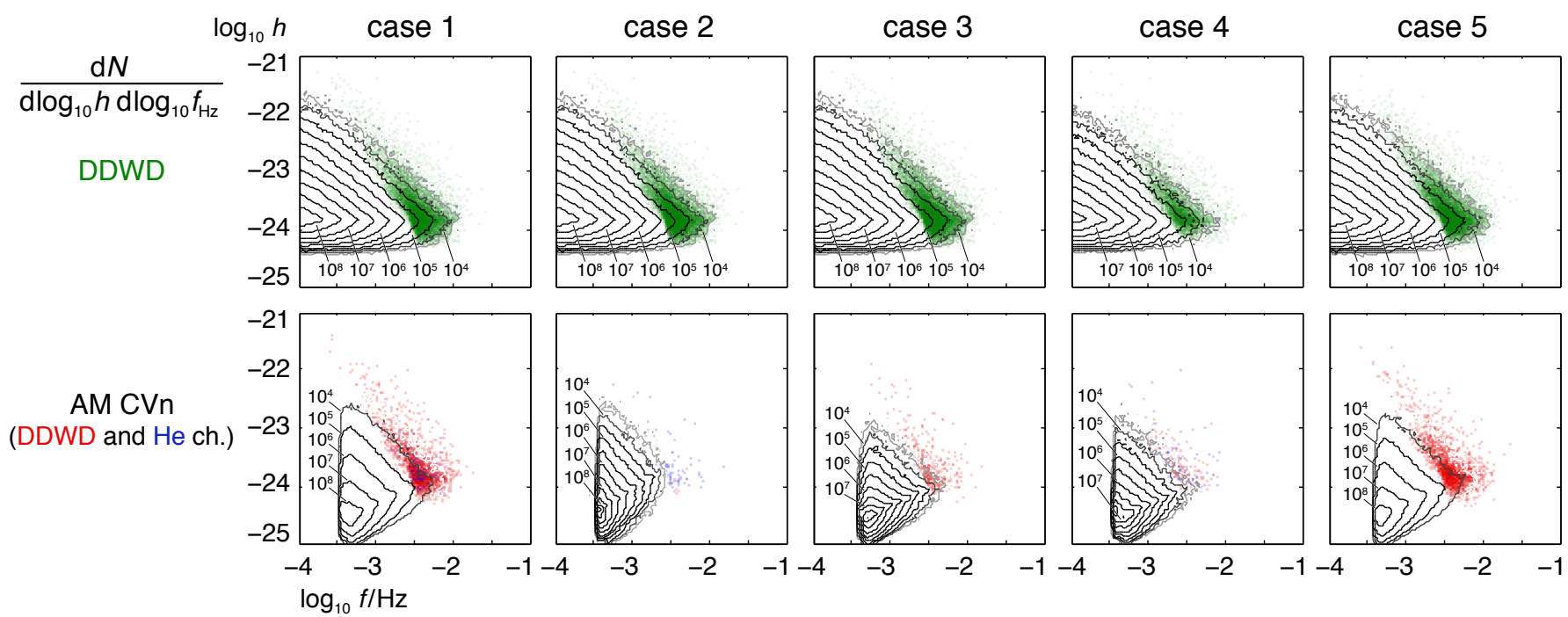

FIG. 1. - Number density as a function of GW-strain amplitude $h$ and GW frequency $f$ for DDWD (top row) and AM CVn (bottom row) systems in our five scenarios. Green dots denote individually detected DDWD systems (for one year of observation with the 5-Mkm detector); red dots AM CVn systems from the DDWD channel, and blue dots AM CVn systems from the He star channel. See Secs. 4.3 and 5.1 for a discussion of individually resolvable sources. The noticeable low-frequency cutoff of AM CVn systems is due to the age of the Universe.

in noise.

\subsection{Simulations of detection and subtraction}

To estimate the number of Galactic-binary systems that we will be able to detect individually with our reference detectors, as well as the confusion noise from the residual unsubtracted systems, we performed simulations of detection and subtraction using a variant of the method introduced by Timpano et al. (2006). To wit:

1. For each of our scenarios we compute the frequency-domain TDI response for each system in the corresponding catalog, using an optimized variant (Vallisneri 2011) of the Mock LISA Data Challenge fast-binary code (Cornish \& Littenberg 2007); we then superpose the responses to produce a full year-long dataset.

2. We run through the catalog and evaluate the SNR of each system (see Sec. B) with respect to a background of instrumental noise plus confusion noise from all nearby systems, estimated as a running median of their power spectral density. We flag as detected the systems with $\mathrm{SNR}>\mathrm{SNR}_{\mathrm{thr}}$.

3. We subtract the signals detected in step 2 from the dataset. We then go back to step 2 and iterate, using the resulting lower estimate of confusion noise.
4. The process converges after a few iterations, with the number of detected systems decreasing better than exponentially. We then evaluate and fit the power spectral density from the residual confusion foreground.

This process represents a rather optimistic model of source detection and subtraction that neglects two important effects: source detection will be confused by the presence of nearby signals beyond what is predicted by the increased noise level, and subtraction will be degraded by the imperfect estimation of parameters in noise (see, e.g., Crowder \& Cornish 2004). Nevertheless, we can adjust the level of optimism by varying our assumptions regarding the duration of observation $T_{\text {obs }}$, the detection threshold $\mathrm{SNR}_{\mathrm{thr}}$, and the number $N_{\text {obs }}$ of TDI observables that are available (two, in effect, for the standard LISA configuration with five or six operating links; one for a two-arm configuration).

It turns out that our results for the number of detected sources and for the residual confusion foregrounds are fit remarkably well by power laws of a single effective-SNR parameter $\rho_{\text {eff }}=\operatorname{SNR}_{\text {thr }}\left(T_{\text {obs }} / \mathrm{yr}\right)^{-1 / 2}\left(N_{\text {obs }}\right)^{-1 / 2}$. As a baseline we take a standard assumption, consistent with prior literature, of one year, one observable (the standard unequal-arm Michelson $X$ ), and $\mathrm{SNR}_{\mathrm{thr}}=5$. A conservative assumption would correspond to taking one year, one observable, and $\mathrm{SNR}_{\text {th }}=10$, yielding $\rho_{\text {eff }}=10$; an 
optimistic assumption to five years, two independent observables, and $\mathrm{SNR}_{\mathrm{th}}=5$, yielding $\rho_{\text {eff }}=1.58$. Results are not sensitive to details such as the choice of runningmedian window, and whether signals are subtracted immediately or after each full catalog run-through.

\section{RESULTS}

In this section we present our results on the impact that different Galactic populations of DDWD and AM CVn systems have on the number of individually detectable sources (Sec. 5.1) and on the total and confusion GW foregrounds (Sec. 5.2), for our two representative spacebased GW detectors and for each of our five population scenarios.

Figure 2 recapitulates the findings of our simulations: it shows the frequency-space density of catalog sources, as well as the fraction of frequency bins with at most one source (bottom panel); the power spectral density of the unsubtracted (blue) and subtracted (red) confusion foreground, compared to the instrument noise (black, all in the middle panel); and the location and numbers of detected sources (dots in the middle panel, histograms in the top panels). The detected sources lie approximately a factor of $\mathrm{SNR}_{\mathrm{th}}^{2}$ above the composite of instrument noise and subtracted confusion noise.

\subsection{Individually detected sources}

Tables 2 (for the $5 \mathrm{Mkm}$ detector) and 3 (for the $1 \mathrm{Mkm}$ detector) show the number of recovered systems of each type in our five scenarios. The numbers quoted correspond to simulation results for one year, one interferometric observable, and $\mathrm{SNR}_{\text {thr }}=5$; the power laws give approximate fits as functions of $\rho_{\text {eff }}=\operatorname{SNR}_{\text {thr }}\left(T_{\text {obs }} / \mathrm{yr}\right)^{-1 / 2}\left(N_{\text {obs }}\right)^{-1 / 2}$.

Consider first the number of detectable sources as seen by the longer, standard-LISA-like detector. In all cases except the "fewer progenitors" scenario 4, the number of detected DDWDs remains comparable to prior optimistic projections $(\sim 12,000-14,000)$. Even in case 4 , where the DDWD systems in the catalog are reduced by a factor of five, detections drop only by half, thanks to weaker confusion. By contrast, the number of detected AM CVn varies considerably depending on their density and formation scenarios $(\sim 15-2,000)$. In cases 2 and 3 , more DDWDs are detectable at relatively high frequencies because of the reduced confusion noise from fewer AM CVn.

Case 1 was chosen to be directly comparable to the standard population catalogs of earlier works Nelemans et al. 2001a b c, 2004), but the claimed number of detections for AM CVn systems is significantly lower than those earlier estimates, derived with a simple resolvability criterion of a single source per frequency bin with instrument-noise SNR > 1 (Nelemans et al. 2001b, 2004). This is unsurprising, given that in our analysis we model detection by identifying and subtracting bright sources iteratively, and that we require a higher SNR, defined with respect to both instrument and confusion noise. Furthermore, in this paper we use proper interferometric observables instead of "raw" GW strain.

Downscaling the detector's armlength to $1 \mathrm{Mkm}$ approximately halves the number of DDWD systems detected for $\rho_{\text {eff }}=5$, except for case 4 , where the decrease is $\sim 3$. The number of AM CVn system detections is likewise reduced by factors $\sim 3-4$. Note however that the $\rho_{\text {eff }}$ scalings of Tables 2 and 3 are slightly different. The ESA eLISA/NGO study (Amaro-Seoane et al. 2012) finds numbers compatible with ours, once we take into account our noise assumptions for the $1 \mathrm{Mkm}$ detector, which are somewhat more favorable than NGO's.

\subsection{Unsubtracted and residual compact-binary foregrounds}

In Appendix A, we apply a familiar analytical treatment of the compact-binary GW foreground to a broad range of Galactic models examined in the literature, and we conclude that the foreground depends strongly on the Galactic binary merger rate and on their characteristic chirp masses, but only weakly on other parameters. Namely,

$$
\begin{aligned}
S_{h}(f) & \simeq 1.9 \times 10^{-44}(f / \mathrm{Hz})^{-7 / 3} \mathrm{~Hz}^{-1} \\
& \times\left(\frac{\mathcal{D}_{\text {char }}}{6.4 \mathrm{kpc}}\right)^{-2}\left(\frac{\mathcal{R}_{\text {gal }}}{0.015 / \mathrm{yr}}\right)\left(\frac{\mathcal{M}_{\text {char }}}{0.35 M_{\odot}}\right)^{5 / 3},
\end{aligned}
$$

where $\mathcal{D}_{\text {char }}$ is the characteristic distance from Earth to Galactic binaries (a weak function of the model); $\mathcal{R}_{\text {gal }}$ is the binary merger rate in the Galaxy; and $\mathcal{M}_{\text {char }}$ is the characteristic chirp mass $\left\langle\mathcal{M}^{5 / 3}\right\rangle^{-5 / 3}$. The $f^{-7 / 3}$ slope describes the power emitted at GW frequency $f$ by a single binary evolving due to GWs, and it applies to a stationary ensemble where new binaries are born with constant rate (equal to $\mathcal{R}_{\text {gal }}$ ) at frequencies below the detector bandwidth. Equation (6) is normalized to values representative of our assumptions; as shown in the left panel of Fig. 3, it is in good agreement with the results of our simulations for cases 1-3 and 5, while the coefficient is reduced by a factor of five for case 4 .

The residual confusion foreground consists of the unresolvable DDWD and AM CVn systems that are left once relatively brighter or isolated sources have been subtracted iteratively from the original population catalog. The residual foregrounds found in our simulations are very similar among cases $1-3$ and 5, while they are a factor of five weaker for case 4 . We note that the overall number of DDWD systems in cases 1-3 and 5 differ by a factor of five, thereby confirming earlier work and our predictions that the DDWD population provides the dominant component to the strength of the confusion foregrounds (both unsubtracted and subtracted). As shown in the middle and right panels of Fig. 3. for both the $5 \mathrm{Mkm}$ and $1 \mathrm{Mkm}$ detectors, the total equivalent strain noise (instrument noise plus confusion foreground) is fit well by the expression

$$
S_{h}^{\text {total }}(f)=S_{h}^{\text {inst }}+S_{h}^{\text {conf }} \times \tanh ^{\alpha}\left(\frac{1}{2} \beta \mathrm{d} N / \mathrm{d} f\right),
$$

where $\alpha \simeq 1, \beta \simeq 1 / \mathrm{yr}$,

$$
S_{h}^{\operatorname{conf}}(f)=1.4 \times 10^{-45}(f / \mathrm{Hz})^{-8 / 3} \mathrm{~Hz}^{-1},
$$

and where

$$
\frac{\mathrm{d} N}{\mathrm{~d} f}=5 \times 10^{-3}(f / \mathrm{Hz})^{-11 / 3} \mathrm{~Hz}^{-1}
$$

is the frequency-domain density of binary systems, which modulates the transition from the confused to the resolved regime. Equation (7) is formulated in analogy to 


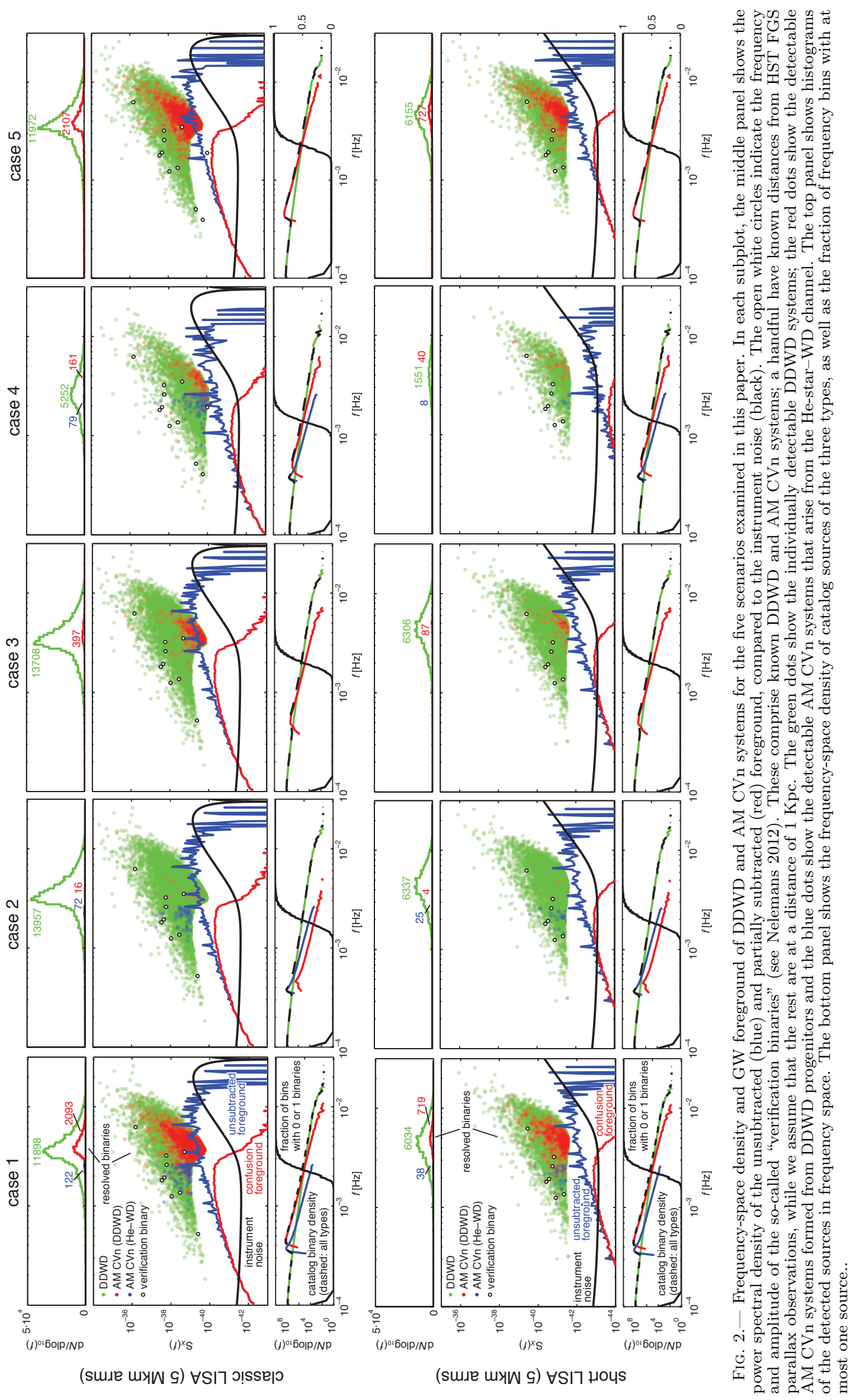


TABLE 2

Detectable sources in our simulations (5 Mkm Detector). Values are Shown for SNR $\mathrm{thr}_{\mathrm{t}}=5$, One interferometric obSERVABLE, AND ONE YeAR OF OBSERVATION, With APPROXimate SCALINGS AS A FUNCTION OF $\rho_{\text {eff }}=\operatorname{SNR}_{\mathrm{thr}}\left(T_{\mathrm{obs}} / \mathrm{yr}\right)^{-1 / 2}\left(N_{\mathrm{obs}}\right)^{-1 / 2}$.

\begin{tabular}{rrrc}
\hline \hline & DDWD & AM CVn (DDWD) & AM CVn $(H e-W D)$ \\
\hline case 1 & $11,898 \times\left(\rho_{\text {eff }} / 5\right)^{-1.4}$ & $2,093 \times\left(\rho_{\text {eff }} / 5\right)^{-1.8}$ & $122 \times\left(\rho_{\text {eff }} / 5\right)^{-2.8}$ \\
case 2 & $13,957 \times\left(\rho_{\text {eff }} / 5\right)^{-1.4}$ & $16 \times\left(\rho_{\text {eff }} / 5\right)^{-1.7}$ & $72 \times\left(\rho_{\text {eff }} / 5\right)^{-2.9}$ \\
case 3 & $13,708 \times\left(\rho_{\text {eff }} / 5\right)^{-1.4}$ & $397 \times\left(\rho_{\text {eff }} / 5\right)^{-2.0}$ & none \\
case 4 & $5,252 \times\left(\rho_{\text {eff }} / 5\right)^{-1.2}$ & $161 \times\left(\rho_{\text {eff }} / 5\right)^{-1.7}$ & $79 \times\left(\rho_{\text {eff }} / 5\right)^{-1.8}$ \\
case 5 & $11,972 \times\left(\rho_{\text {eff }} / 5\right)^{-1.4}$ & $2,107 \times\left(\rho_{\text {eff }} / 5\right)^{-1.7}$ & none \\
\hline \hline
\end{tabular}

TABLE 3

Detectable sources in our simulations (1 Mkm Detector). Values are Shown for SNR $\mathrm{thr}_{\mathrm{t}}=5$, ONe interferometric

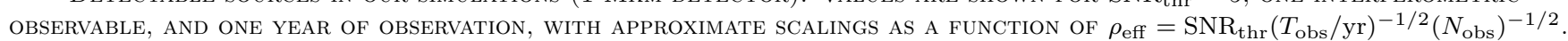

\begin{tabular}{ccrc}
\hline \hline & DDWD & AM CVn (DDWD) & AM CVn $(\mathrm{He}-W D)$ \\
\hline case 1 & $6,034 \times\left(\rho_{\text {eff }} / 5\right)^{-1.2}$ & $719 \times\left(\rho_{\text {eff }} / 5\right)^{-1.4}$ & $38 \times\left(\rho_{\text {eff }} / 5\right)^{-2.6}$ \\
case 2 & $6,337 \times\left(\rho_{\text {eff }} / 5\right)^{-1.2}$ & $4 \times\left(\rho_{\text {eff }} / 5\right)^{-1.4}$ & $25 \times\left(\rho_{\text {eff }} / 5\right)^{-2.2}$ \\
case 3 & $6,306 \times\left(\rho_{\text {eff }} / 5\right)^{-1.2}$ & $87 \times\left(\rho_{\text {eff }} / 5\right)^{-1.8}$ & none \\
case 4 & $1,551 \times\left(\rho_{\text {eff }} / 5\right)^{-1.1}$ & $40 \times\left(\rho_{\text {eff }} / 5\right)^{-1.3}$ & $8 \times\left(\rho_{\text {eff }} / 5\right)^{-2.2}$ \\
case 5 & $6,155 \times\left(\rho_{\text {eff }} / 5\right)^{-1.2}$ & $727 \times\left(\rho_{\text {eff }} / 5\right)^{-1.4}$ & none \\
\hline \hline
\end{tabular}
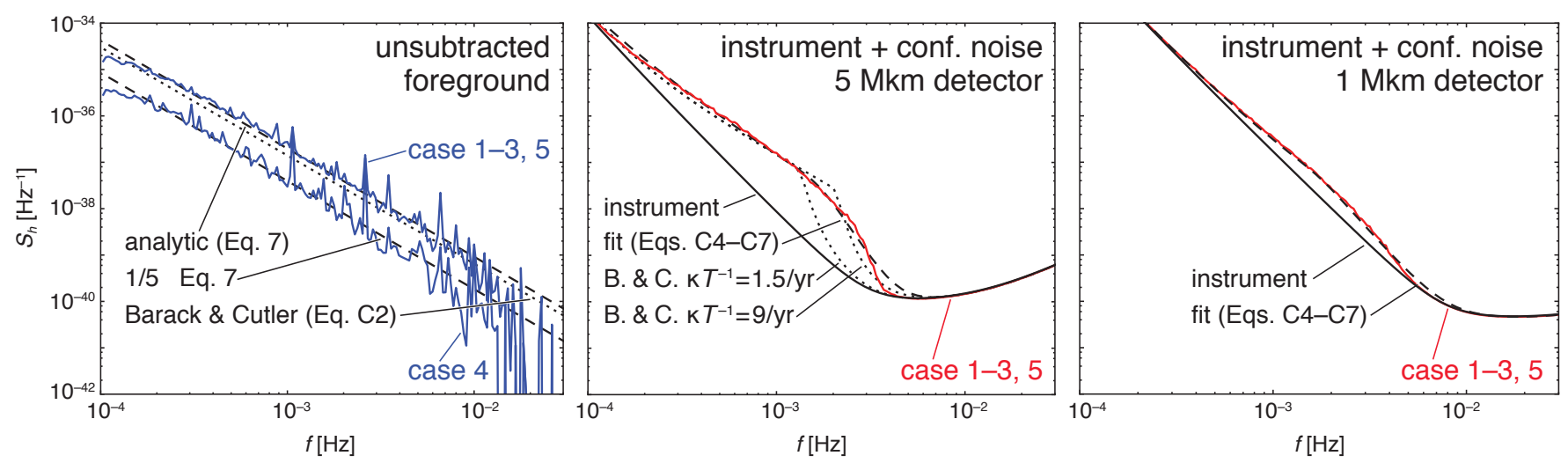

FIG. 3. - Left panel: power spectral density of GW strain for the unsubtracted Galactic foreground, as measured in our simulations for cases 1-3 and 5 (top continuous curve) and case 4 (bottom continuous curve); the foreground is fit well by our Eq. 6 , with a $1 / 5$ smaller coefficient for case 4 (dashed curves). For comparison, the dotted curves shows the Barack and Cutler estimate Eq. C22. At high frequencies, the simulated spectra drop because frequency bins are unevenly occupied, and because of the onset of mass transfer; at low frequency, they drop because some binaries are born at frequencies within the detector's bandwidth, contrary to the assumptions that yield the $f^{-7 / 3}$ profile. [These spectra of GW strain do not include the instrument response to GWs; to compare with the TDI $X$ spectra of Fig. 2 one would multiply by the frequency-dependent response of Eq. (C8).] Middle and right panels: total equivalent GW-strain noise (instrument noise plus residual Galactic-binary confusion noise) for the 5-Mkm and 1-Mkm detectors of our study, as obtained in our simulations for cases $1-3$ and 5 and for $\rho_{\text {eff }}=5$ (red curves); case 4 is not shown to avoid visual clutter. The total noise is fit well (dashed curves) by our expressions Eqs. (7)-99 [see also Eqs. [C4 - C77], and less well (dotted curves) by Barack and Cutler's standard prescription Eqs. C1 - C3, which improves by setting $\kappa T^{-1} \simeq 9 / \mathrm{yr}$. 
the standard expression Eq. C1) [see (Barack \& Cutler $2004 \mathrm{a}$ b)], with a different functional form that fits our simulations better. In App.C we give more precise $\alpha$ and $\beta$ fits as functions of $\rho_{\text {eff }}$, and we provide expressions for the total TDI $X$ noise and for the seasonal modulation of the GW foreground due to detector motion (Giampieri \& Polnarev 1997, Seto 2004, Edlund et al. 2005).

\section{CONCLUSIONS}

In this work, we have examined the impact that different observationally inferred populations of Galactic white dwarf binaries have on the number of individually detectable sources and on the confusion foreground for future low-frequency GW observations. Earlier studies of Galactic white dwarf binaries as GW sources relied primarily on population synthesis, because of the small number of observed systems, in particular mass transferring ones (i.e., AM CVn systems). However, the past several years have seen a flurry of new observations, tripling the known detached systems that will merge within a Hubble time, doubling the known AM CVn systems, and leading to new, lower estimates for the local densities of the latter.

To understand the consequences of these new estimates on GW detection, we have generated a range of plausible populations of white dwarf binaries, assuming different $\mathrm{AM} \mathrm{CVn}$ formation scenarios. For example, in one model (case 3) we postulate that fewer AM CVn systems are observed by SDSS than predicted because the tidal coupling between the spin of the accretor and the orbit is not as efficient as expected, so more systems are lost to mergers. We have then investigated the resulting numbers of individually resolvable GW sources and the magnitude of the total and residual GW foregrounds.

In four out of five models, we find that the number of detectable detached systems is $\sim 12,000-14,000$, for one year of observation by a LISA-like GW detector with 5 Mkm arms (using an optimistic detection threshold, but considering a single interferometric observable). These four models leave unchanged the number of detached binaries used in earlier estimates, but make various assumptions about the number of systems lost to mergers or to edge-lit detonations, or (in case 5) about the spatial distribution of binaries. In one model (case 4 ), which instead explains the observed local AM CVn density with five times fewer detached progenitor systems, we find that $\sim 6,000$ of the latter should be detectable. For the $1-\mathrm{Mkm}$-arm detector, detections would be $\sim 6,000$ and 1,500 , respectively. The number of detectable $A M C V n$ systems ranges from several tens to a few hundred (for cases 2-4), or even as high $\sim 2,000$ (for cases 1 and 5), again for the $5-\mathrm{Mkm}$ detector. For the $1-\mathrm{Mkm}$ detector these numbers are smaller by approximately a factor four. See Tables 2 and 3 for details.

Now, why is it that even in our upper-bound model (case 1), where the total number of AM CVn systems is comparable to earlier estimates, we project far fewer AM CVn detections than the 10,000 quoted by Nelemans et al. (2001b, 2004)? Conversely, in four out of five models we project more detached-binary detections $(12,000$ 14,000 vs. 10,000) than Nelemans et al. (2001b). Such differences arise because we define detection by way of an iterative identify-and-subtract process, where a source's signal-to-noise ratio is evaluated with respect to noise from both the instrument and the partially subtracted foreground. Our approach is similar to that used by Timpano et al. (2006). By contrast, according to Nelemans et al. (2001b, 2004), a source is detectable if it has a signal-to-noise ratio (relative to instrument noise alone) greater than five, or a signal-to-noise ratio greater than one with no other source in the same frequency bin. Our more stringent definition impacts the number of detected AM CVn systems more than their detached counterparts at the same frequencies, because AM CVn systems have smaller chirp masses, and therefore relatively weaker GW-strain amplitudes.

Indeed, we confirm that the dominant contribution to the diffuse $G W$ foreground comes from detached binaries, which have larger chirp masses. In App. A, we show that the spectrum of the unsubtracted foreground can be derived robustly by simple analytical arguments, and depends mainly on the Galactic merger rate and the characteristic binary chirp mass [see Eq. [6]]. These analytical estimates agree well with our numerical results. In addition, for both the $5 \mathrm{Mkm}$ and $1 \mathrm{Mkm}$ detectors we provide fitting expressions [Eqs. (7)-(9)] for the residual confusion foreground due to unresolvable sources. Our estimates are comparable to (but measurably different from) the standard formulas discussed by Barack \& Cutler (2004b) and used by many authors in the past.

The projected detection numbers and residual confusion foregrounds change significantly if we use different assumptions on the detection threshold, the duration of observation, and the number of available interferometric observables. We have simulated the identify-and-subtract process for several such assumptions, and we find empirically that the detection numbers and confusion-foreground fitting parameters scale as power laws of a single effective threshold $\rho_{\text {eff }}=$ $\mathrm{SNR}_{\text {thr }}\left(T_{\text {obs }} / \mathrm{yr}\right)^{-1 / 2}\left(N_{\text {obs }}\right)^{-1 / 2}$.

In one of our models (case 5), we have examined (to our knowledge, for the first time) how GW observations would change if we modify the evolution and structure of the Galactic disk to match the observed local AM CVn density, while keeping overall binary numbers as in case 1. We find no significant impact on the number of detectable systems, nor on the strength or shape of the confusion foreground. However, on average the detected systems will have measurably smaller GW amplitudes, because of their larger distances from the Solar system. We conjecture that such distance measurements could be used to infer the structure of the Galactic disk.

Finally, we expect that the advent of wide-field synoptic and targeted surveys (such as the Palomar Transient Factory and the ELM survey respectively), as well as ESA's GAIA mission, will continue to provide new data to this field of research, and will enable more stringent constraints on binary populations. So far, the observed density of detached systems appears to be consistent with theoretical estimates, but a detailed population analysis based on the ELM survey has yet to be undertaken. Furthermore, all estimates of AM CVn densities based on surveys are made uncertain by the intricate selection effects. Because of these uncertainties, in this paper we chose to examine a variety of population models that span the range of likely outcomes. Our overall conclusion is that the Galactic-binary science enabled by low- 
TABLE 4

Estimates of GW from Populations of Compact Binaries

\begin{tabular}{|c|c|c|c|}
\hline reference & model & source classes & input population \\
\hline this work, Nelemans et al. (2004) & $\mathrm{RS}+\mathrm{CB}$ & DDWD + AMCVn & $\mathrm{SeBa}^{1}$ \\
\hline Nelemans et al. $(2001 \mathrm{~b})$ & $\mathrm{RS}+\mathrm{CB}$ & DDWD + AMCVn & $\mathrm{SeBa}^{1}$ \\
\hline Edlund et al. $(2005)$ & $\mathrm{RS}+\mathrm{CB}$ & DDWD + AMCVn & $\mathrm{SeBa}^{1}$ \\
\hline Timpano et al. $(2006)$ & $\overline{\mathrm{RS}+\mathrm{CB}}$ & DDWD & SeBa ${ }^{1}$, Webbink $(1984)$ \\
\hline$\overline{\text { Seto }}(\overline{2002})$ & $\mathrm{RS}+\mathrm{CB}$ & DDWD & Webbink $(\overline{1984})^{-}$ \\
\hline Bender \& Hils $(1997)$ & $\mathrm{RS}+\mathrm{CB}$ & DDWD & Webbink \\
\hline Hils et al. $(1990)$ & $\mathrm{CB}$ & DDWD & Webbink $\sqrt{1984}$ \\
\hline Hils \& Bender $(2000)$ & CB & AMCVn & Iben \& Tutukov (1984); Tutukov \& Yungelson (1996) \\
\hline Hils 1998$)$ & $\mathrm{RS}+\mathrm{CB}$ & $\mathrm{AMCVn}$ & Iben \& Tutukov (1984); Tutukov \& Yungelson (1996) \\
\hline Yu \& Jeffery $(2010)$ & $\overline{\mathrm{RS}+\mathrm{CB}}$ & DDWD (+ halo) & $\mathrm{BSE}^{2}+\operatorname{Han}(1998)$ \\
\hline Liu $(2009)$; Liu et al. $(2010)$ & $\mathrm{RS}+\mathrm{CB}$ & DDWD & $\mathrm{BSE}^{2}+\overline{\operatorname{Han}}(\overline{1998})$ \\
\hline Farmer \& Phinney $(2003)$ & $\mathrm{CB}$ & extragalactic DDWD & $\mathrm{BSE}^{2}$ \\
\hline Webbink \& Han $(19 \overline{98})$ & $\mathrm{CB}$ & DDWD & Han 1998 \\
\hline $\left.\begin{array}{l}\text { Ruiter et al. } \\
\text { Ruiter et al. }(\overline{2010} \\
2009\end{array}\right)$ & $\overline{\mathrm{RS}+\mathrm{CB}}$ & $\begin{array}{l}\begin{array}{l}\text { DDWD + AMCVn } \\
\text { (halo) }\end{array} \\
\end{array}$ & StarTrack, Belczynski et al. 2002,2008 \\
\hline Benacquista et al. (2004) & $\mathrm{RB}+\mathrm{CB}$ & DDWD (+ globular) & Specified in paper \\
\hline Postnov \& Prokhorov (1998) & $\mathrm{CB}$ & DDWD & See Lipunov et al. (1996) \\
\hline Lipunov et al. $(1995)$ & $\mathrm{CB}$ & DDWD (+ halo) & specified in paper \\
\hline Lipunov \& Postnov (1987) & $\mathrm{CB}$ & compact binaries & specified in paper \\
\hline Lipunov et al. (1987) & & & \\
\hline Giampieri \& Polnarev (1997) & $\mathrm{CB}$ & generic & generic \\
\hline Evans et al. (1987) & $\mathrm{CB}$ & DDWD & specified in paper \\
\hline \multicolumn{4}{|c|}{ 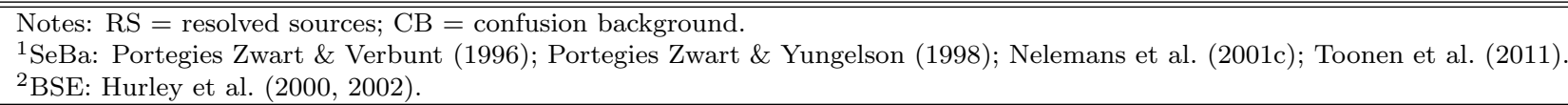 } \\
\hline
\end{tabular}

frequency GW detectors is very robust, for a broad range of possible binary populations and detector sensitivities.

Acknowledgments. For useful discussions and interactions, we are grateful to Lars Bildsten, Roseanne Di Stefano, Paul Groot, Mukremin Kilic, Shri Kulkarni, David Levitan, Avi Loeb, Sterl Phinney, Tony Piro, and Ken Shen. Part of this work was performed by TAP while at the Aspen Center for Physics, which is supported by NSF Grant \#1066293. GN is supported by NWO and FOM. MV is grateful for support from the LISA Mission Science Office. Part of this work was performed at the Jet Propulsion Laboratory, California Institute of Technology, under contract with the National Aeronautics and Space Administration. Government sponsorship acknowledged. Copyright 2011.

\section{APPENDIX}

\section{A. PREVIOUS ESTIMATES OF GW EMISSION FROM GALACTIC BINARIES}

More than two dozen papers have made estimates of the GW signal from individually detectable Galactic compact binaries and from the continuum of unresolvable sources at low frequencies (variously called "confusion background" or "Galactic foreground"). An earlier informative review of the literature is given by Ruiter et al. (2010).

Table 4 compares a subset (large, but possibly not complete) of relevant published estimates, grouped by population model. For each entry, we show whether the estimates include resolved sources (RS), the continuum background (CB), or both; we show also which classes of binaries are included. In Table 5 we provide relevant scale lengths for the Galactic disk and bulge distributions, where available; these are indicative of the range of assumed Galactic models, but they do not provide a complete picture since different density laws also appear. We do not give quantitative information on possible halo components, which are also frequently used. The major differences between the various estimates arise from two sets of assumptions:

1. Assumptions about the relative numbers of systems in terms of total mass, mass ratio, and separation. These arise from different treatments of compact-binary progenitor evolution and of the stability of mass-transferring systems. Approaches include both analytic estimates and Monte Carlo population-synthesis calculations.

2. Assumptions about Galactic structure and population space densities. These can affect estimates in two ways: first, the magnitude of GW signals will depend on the scale size assumed for the Galactic population; second, the "calibration" of the local number density of sources using observations of a particular source type will depend sensitively on the assumptions about Galactic structure.

We now present a semi-analytical derivation of the amplitude and shape of the GW continuum. Similar models have been used extensively in GW data analysis, see for example works by Hughes (2002); Barack \& Cutler (2004a b). Our 
TABLE 5

Estimates of GW from Populations of Compact Binaries - Spatial Distributions

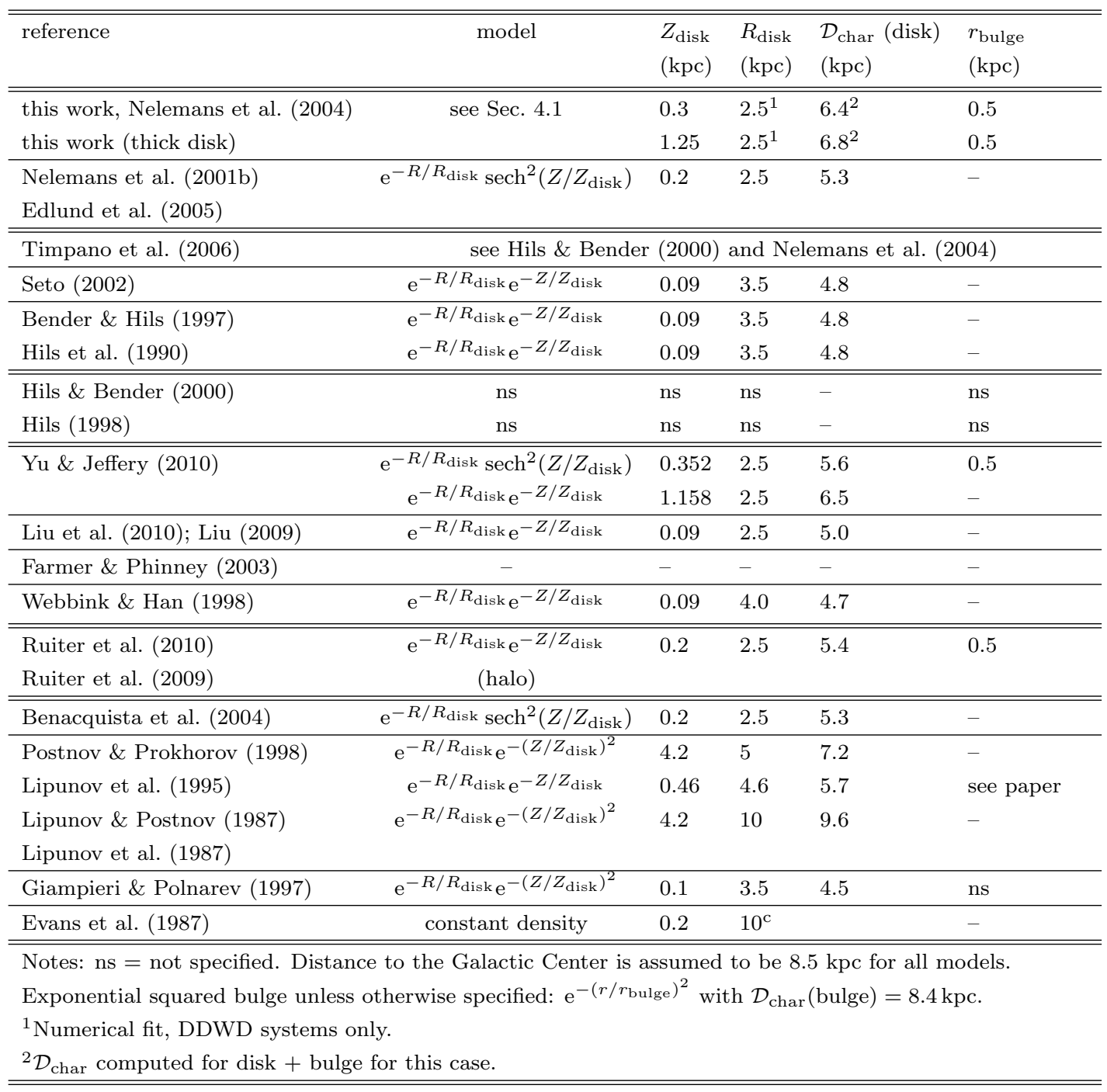

results show that the magnitude and spectral shape of the continuum are a robust function of the merger rate and characteristic chirp mass of Galactic binary systems, and depend only weakly on Galactic structure.

We follow the arguments presented in Evans et al. (1987) and Giampieri \& Polnarev (1997). The GW luminosity of a circular binary and the change in its orbital energy as a function of GW frequency $f=2 f_{\text {orb }}$ are given by $($ Peters $\&$ Mathews 1963)

$$
\frac{d E}{d t}=\frac{32}{5} \pi^{10 / 3} \frac{G^{7 / 3}}{c^{5}} \mathcal{M}^{10 / 3} f^{10 / 3}, \quad \frac{d E}{d f}=\frac{1}{3} \pi^{2 / 3} G^{2 / 3} \mathcal{M}^{5 / 3} f^{-1 / 3} ;
$$

where $E$ is the energy of the system and $f$ is the GW frequency. Combining these expressions yields the frequency evolution of the system,

$$
\frac{d f}{d t}=\frac{96}{5} \pi^{8 / 3} \frac{G^{5 / 3}}{c^{5}} \mathcal{M}^{5 / 3} f^{11 / 3} .
$$

If $\mathcal{R}_{\text {birth }}(f, \mathbf{r})$ is the birth rate per unit frequency and volume of systems with $f_{\text {orb }}<f / 2$, in a stationary regime the density of sources must be

$$
\frac{d^{2} N}{d f d \mathbf{r}}=\frac{\mathcal{R}_{\mathrm{birth}}(f, \mathbf{r})}{d f / d t}=\frac{5}{96} \pi^{-8 / 3} \frac{c^{5}}{G^{5 / 3}} \mathcal{M}^{-5 / 3} \mathcal{R}_{\mathrm{birth}}(f, \mathbf{r}) f^{-11 / 3} .
$$

By continuity, $\mathcal{R}_{\text {birth }}(f, \mathbf{r})$ must also be equal to the merger rate, $\mathcal{R}_{\operatorname{mrg}}(f, \mathbf{r})$, of systems which merge at $f_{\text {orb }}>f / 2$. DDWD systems, which generate most of the GW foreground, merge at $f>10^{-2} \mathrm{~Hz}$, and most of them are born below 
a few $10^{-4} \mathrm{~Hz}$ (although estimates vary). Thus, if we restrict Eq. (A3) between those two frequencies, we may just set $\mathcal{R}_{\text {birth }}(f, \mathbf{r})=\mathcal{R}_{\text {mrg }}(\mathbf{r})$, defined as the total merger rate above $10^{-2} \mathrm{~Hz}$.

Now, the direction-averaged GW strain from a circular binary is given by

$$
h^{2}=\left\langle h_{+}^{2}+h_{\times}^{2}\right\rangle=\frac{1}{\pi^{2}} \frac{1}{d^{2}} \frac{G}{c^{3}} f^{-2} \frac{d E}{d t}=\frac{32}{5} \pi^{4 / 3} \frac{G^{10 / 3}}{c^{8}} \mathcal{M}^{10 / 3} f^{4 / 3}
$$

see for instance (Giampieri \& Polnarev 1997), where $d$ is the luminosity distance to the binary; the corresponding detector response is

$$
h_{I}^{2}=\left\langle h_{+}^{2} F_{+}^{2}+h_{\times}^{2} F_{\times}^{2}\right\rangle \equiv h^{2} F^{2}(\hat{\mathbf{r}}, t),
$$

where $F_{+}$and $F_{\times}$are the antenna-pattern functions (see, e.g., Maggiore 2007) and $F^{2}(\hat{\mathbf{r}}, t)$ is the polarization- and inclination-averaged antenna pattern $\left(\left\langle F_{+}^{2}\right\rangle+\left\langle F_{\times}^{2}\right\rangle\right) / 2$ in the direction $\hat{\mathbf{r}}$.

We combine Eqs. A3, A4, and A5 to obtain the one-sided detector response density $S_{h_{I}}$. The yearly orbital motion of LISA-like detectors modulates the amplitude of signals to individual sources; this effect averages out for isotropic source distributions, but not for the anisotropic Galactic-binary foreground (Giampieri \& Polnarev 1997, Edlund et al. 2005). We therefore average over a year (while assuming for now that all binaries have the same chirp mass $\mathcal{M}$ ), to obtain

$$
\begin{aligned}
S_{h_{I}}(f) & =\left\langle\int h^{2}(d(\mathbf{r}), f) F^{2}(\hat{\mathbf{r}}, t) \frac{d^{2} N}{d f d \mathbf{r}} d \mathbf{r}\right\rangle_{t} \\
& =\frac{1}{3} \pi^{-4 / 3} \frac{G^{5 / 3}}{c^{3}} \mathcal{M}^{5 / 3} f^{-7 / 3} \int \frac{\left\langle F^{2}(\hat{\mathbf{r}}, t)\right\rangle_{t} \mathcal{R}_{\mathrm{mrg}}(\mathbf{r})}{d(\mathbf{r})^{2}} d \mathbf{r} \\
& \equiv \frac{1}{3} \pi^{-4 / 3} \frac{G^{5 / 3}}{c^{3}} \mathcal{M}^{5 / 3} f^{-7 / 3}\left\langle F^{2}\right\rangle_{\hat{\mathbf{r}}, t}\left\langle\mathcal{R}_{\mathrm{mrg}} / d^{2}\right\rangle_{\mathbf{r}}
\end{aligned}
$$

Of the two quantities defined in the last line of Eq. $(\mathrm{A} 6),\left\langle F^{2}\right\rangle_{\hat{\mathbf{r}}, t}$ accounts for the time-, merger-rate- and directionaveraged instrument response, and it depends on the orbit and geometry of the detector. For an isotropic distribution of sources and a triangular interferometer with $60^{\circ}$ angles, $F^{2}(\hat{\mathbf{r}}, t)=1 / 5 \times \sin ^{2}\left(60^{\circ}\right)=3 / 20$, which is often taken as the average isotropic response for LISA-like detectors (Giampieri \& Polnarev 1997). The factor $1 / 5$ is the average of $\left(\left\langle F_{+}^{2}\right\rangle+\left\langle F_{\times}^{2}\right\rangle\right) / 2$ over source locations and polarizations (again, e.g., in Maggiore 2007). Again for a LISA-like detector, but for typical disk populations, $F^{2}(\hat{\mathbf{r}}, t)$ is found empirically to vary by $\sim 10 \%$ from $3 / 20$.

The quantity $\left\langle\mathcal{R}_{\mathrm{mrg}} / d^{2}\right\rangle_{\mathbf{r}}$ is the merger-rate-averaged inverse squared distance. For a Galactic disk, it depends on the scale height and radial scale length, and of course on the position of the detector within the disk. A typical disk density distribution considered in many papers is the squared-hyperbolic-secant disk model,

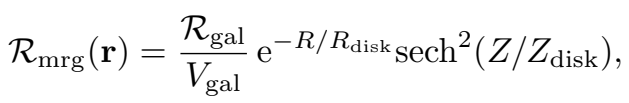

where $\mathcal{R}_{\text {gal }}$ is the total merger rate in the Galaxy and $V_{\text {gal }}$ is the volume of the Galaxy. We find empirically that $\left\langle\mathcal{R}_{\mathrm{mrg}} / d^{2}\right\rangle_{\mathbf{r}}$ varies slowly for $2.5 \mathrm{kpc} \leq R_{\text {disk }} \leq 10 \mathrm{kpc}$ and $0.2 \mathrm{kpc} \leq Z_{\text {disk }} \leq 1 \mathrm{kpc}$. Table 5 summarizes the wide range of Galactic models used in the literature. For most disk models the characteristic distance to Galactic sources $\mathcal{D}_{\text {char }} \equiv\left(V_{\text {gal }} / \mathcal{R}_{\text {gal }}\right)^{-1 / 2}\left\langle\mathcal{R}_{\text {mrg }} / d^{2}\right\rangle^{-1 / 2}$ is approximately $5 \mathrm{kpc}$, and $\mathcal{D}_{\text {char }}+R_{\text {disk }}$ is close to $8.5 \mathrm{kpc}$, the assumed distance to the Galactic center.

Inserting numerical values appropriate for our simulations (cases 1-3) in Eq. A6 yields

$$
S_{h_{I}}(f) \simeq 1.9 \times 10^{-44}(f / \mathrm{Hz})^{-7 / 3} \mathrm{~Hz}^{-1} \times\left\langle F^{2}\right\rangle_{\hat{\mathbf{r}}, t} \times\left(\frac{\mathcal{D}_{\mathrm{char}}}{6.4 \mathrm{kpc}}\right)^{-2}\left(\frac{\mathcal{R}_{\mathrm{gal}}}{0.015 / \mathrm{yr}}\right)\left(\frac{\mathcal{M}_{\mathrm{char}}}{0.35 M_{\odot}}\right)^{5 / 3},
$$

where $\mathcal{M}_{\text {char }}$ is the characteristic chirp mass $\left\langle\mathcal{M}^{5 / 3}\right\rangle^{-5 / 3}$. The power spectral density $S_{h}(f)$ of the GW strain is obtained by dropping the factor $\left\langle F^{2}\right\rangle_{\hat{\mathbf{r}}, t}$ (which is close to $3 / 20$ for LISA-like detector). Equation A8 is in good agreement with the results of our simulations. Note however that the spectra of Fig. 2 are plotted for the TDI observable $X$ (rather than for strain $h$ or strain response $h_{I}$ ), so they must be compared with

$$
S_{X}(f)=\frac{16 x^{2} \sin ^{2} x}{1+0.6 x^{2}} S_{h_{I}}(f)
$$

where $x=2 \pi f L / c$, and $L$ is the detector armlength.

Our analysis indicates that $S_{h_{I}}(f)$ and $S_{h}(f)$ are relatively insensitive to the structure of the Galactic disk, consistent with the results of our simulations, and instead depend strongly on the merger rate $\mathcal{R}_{\text {gal }}$ and the characteristic chirp mass $\mathcal{M}_{\text {char }}$. By constrast, we expect that the local population density of sources, as well as the number of resolvable source as a function of their GW strain, will depend roughly linearly on the disk's scale height.

The transition between the confused and resolved detection regimes is characterized by $d N / d f$, which is determined by the evolution of the chirp frequency and by the absolute merger-rate density of sources. Integrating Eq. A3 over 
$\mathbf{r}$ we find

$$
\frac{d N}{d f}=5 \times 10^{-3}(f / \mathrm{Hz})^{-11 / 3} \mathrm{~Hz}^{-1} \times\left(\frac{\mathcal{R}_{\mathrm{gal}}}{0.015 / \mathrm{yr}}\right)\left(\frac{\mathcal{M}_{\mathrm{char}}}{0.35 M_{\odot}}\right)^{-5 / 3}
$$

consistent with our simulations.

This simple analytical derivation of the GW continuum shows that the major differences between the various estimates listed in Table 4 arise primarily from two sources: the total merger rate of systems $\mathcal{R}_{\text {gal }}$ and the composition of the source population, leading to a different characteristic chirp mass $\mathcal{M}_{\text {char }}$. $\mathcal{D}_{\text {char }}$ is also relevant, but less important. (Remember however that our derivation is only predictive for frequencies high enough that most binaries form at longer periods - for LISA-like detectors, this is true above $10^{-4} \mathrm{~Hz}$. For lower frequencies, the shape of the continuum depends on the detailed accounting of birth rate vs. period.)

\section{B. LOW-FREQUENCY GW INTERFEROMETERS AND THEIR RESPONSE TO BINARY WAVES}

LISA (Prince et al. 2009, Jennrich 2009), the space-based GW observatory studied jointly by NASA and ESA until early 2011, comprises three spacecraft orbiting the Sun in a quasi-equilateral configuration with armlengths $\sim 5 \times 10^{6} \mathrm{~km}$. LISA measures GW strain by monitoring the oscillating distance between freely-falling test masses, using heterodyne laser interferometry. The spacecraft hover around the test masses to protect them from external perturbations, performing slight orbital corrections with extremely precise micro-Newton thrusters.

Although LISA is sometimes described as a large Michelson interferometer in space, the lasers exchanged by spacecraft are neither split nor reflected. Rather, two lasers on each spacecraft are used to establish six one-way links; each link provides a test-mass-referenced interferometric measurement $y_{i j}$ of the phase (or equivalently, the frequency) of the incoming laser, compared to the local, outgoing beam. In terms of fractional laser frequency, the effect of a plane GW on the links is given by the two-pulse response (Estabrook \& Wahlquist 1975)

$$
y_{i j}(t)=\frac{1}{2} \frac{\hat{n}_{i j} \cdot\left[\mathrm{h}\left(p_{i}, t-L_{i j}\right)-\mathrm{h}\left(p_{j}, t\right)\right] \cdot \hat{n}_{i j}}{1-\hat{n}_{i j} \cdot \hat{k}},
$$

where $\hat{n}_{i j}^{i}$ is the unit vector from spacecraft $i$ to $j ; \hat{k}$ the GW propagation vector; $\mathrm{h}\left(p_{k}, t\right)$ the TT-gauge GW strain (see, e.g., Maggiore 2007) at the spacecraft position $p_{k}$ and at time $t$; and $L_{i j}$ the photon time of flight along the arm. Thus, a GW pulse is registered twice in $y_{i j}$ : once when it impinges on spacecraft $j$, and a time $L_{i j}$ after it has impinged on spacecraft $i$. In the limit of long GW wavelengths $\lambda \gg L$, the $y_{i j}$ response to plane GWs is therefore proportional to L.

Each $y_{i j}$ measurement is also affected by displacement noise from residual test-mass accelerations, position noise from optical-path and shot noise in the heterodyne measurements, and, most importantly, by oscillations in the central laser frequency, which are many orders of magnitude stronger than expected GW imprints, and which appear as $y_{i j}=C_{j i}(t)-C_{i j}\left(t-L_{i j}\right)$, with $C_{i j}$ the frequency noises for the six lasers. Laser frequency noise is removed by combining multiple one-way measurements with appropriate time delays so that each laser appears in canceling pairs, as in the unequal-arm Michelson observable

$$
\begin{aligned}
X(t) & =\left[y_{31}(t)+y_{13}\left(t-L_{31}\right)+y_{21}\left(t-L_{31}-L_{13}\right)+y_{12}\left(t-L_{31}-L_{13}-L_{21}\right)\right] \\
& -\left[y_{21}(t)+y_{12}\left(t-L_{21}\right)+y_{31}\left(t-L_{21}-L_{12}\right)+y_{13}\left(t-L_{21}-L_{12}-L_{31}\right)\right] .
\end{aligned}
$$

This noise-suppression technique, specific to LISA-like interferometers, is known as time-delay interferometry (TDI; see, e.g., Vallisneri 2005 for a review). TDI observables can be seen as synthesized interferometers, since their $y_{i j}$ components trace the paths (and reproduce the phase delays) of light across recognizable interferometric configurations (hence X's "Michelson" designation). Many different observables are possible with LISA's six laser links, but all can be reconstructed from a set of three observables, such as the unequal-arm Michelson $X, Y$, and $Z$ centered on the three spacecraft (Vallisneri et al. 2008).

In this paper, we assume an rms displacement noise

$$
S_{\mathrm{pm}}^{1 / 2}(f)=3 \times 10^{-15} \mathrm{~ms}^{-2} \mathrm{~Hz}^{-1 / 2} \times \sqrt{1+\left(\frac{f}{10^{-4} \mathrm{~Hz}}\right)^{-1}} \times \sqrt{1+\left(\frac{f}{0.008 \mathrm{~Hz}}\right)^{4}}
$$

for each of the proof masses, and a total rms position noise

$$
S_{\mathrm{pm}}^{1 / 2}(f)=18 \times 10^{-12} \mathrm{~m} \mathrm{~Hz}^{-1 / 2} \times \sqrt{1+\left(\frac{f}{0.002 \mathrm{~Hz}}\right)^{-4}}
$$

for each $y_{i j}$ measurement.

The GW mission currently under study as an ESA-led project (Amaro-Seoane et al. 2012) would decrease cost primarily by reducing spacecraft and propellant mass, allowing for launch on smaller rockets than envisaged for LISA. Propellant is saved by placing the spacecraft closer together and closer to Earth. At the low-frequency end, the shorter armlengths reduce the response to GWs proportionally; for the same displacement noise, sensitivity also decreases by the same ratio. At the high-frequency end, the laser power available for position measurement increases as $L^{-2}$, since 
beams are broadly defocused at millions of kms, improving shot noise (but not other optical noises) by an rms factor $L^{-1}$. As a consequence, the sweet spot of the LISA sensitivity shifts to higher frequencies.

Spacecraft mass may also be saved by adopting a two-arm "mother-daughters" configuration with only four laser links, two each between the middle "mother" spacecraft and one of the "daughters". With four links, only one independent TDI observable can be formed, with a loss in sensitivity averaging $\sqrt{2}$ at compact-binary frequencies. Because no trade studies have yet been performed to optimize the payload for the new orbits, in this paper we consider a "short LISA" configuration with aggressively reduced $1 \times 10^{6}-\mathrm{km}$ armlengths, and with the LISA displacement and position noises (except for the power rescaling of the position-noise component, which we assume as five times smaller in $\mathrm{rms}$ ). The actual sensitivity of a cost-constrained space-based GW observatory is likely to fall somewhere between this short LISA and the classic configuration.

Searches for GWs of known shape proceed by correlating detector data with theoretical waveform templates computed for the expected range of source parameters. The statistical confidence of detection for a $\mathrm{GW}$ signal $h$ immersed in Gaussian additive noise $n$ is described by the signal-to-noise ratio (SNR) of the data $s=h+n$ after filtering by the best-fitting normalized template $\hat{t}$. For a single TDI observable $X$, this is given by (Cutler \& Flanagan 1994)

$$
\rho(s ; \hat{t})=4 \operatorname{Re} \int_{0}^{\infty} \frac{\tilde{X}_{s}(f)^{*} \tilde{X}_{\hat{t}}(f)}{S_{X}(f)} \mathrm{d} f,
$$

where $\tilde{X}_{s}(f)$ and $\tilde{X}_{\hat{t}}(f)$ are the Fourier transforms of the TDI data and of the TDI response to waveform $\hat{t}$ respectively; "** denotes the complex conjugate; and $S_{X}(f)$ is the noise power spectral density of TDI observable $X$. Normalization amounts to requiring $\rho(\hat{t} ; \hat{t})=1$.

With this noise weighting, the false-alarm probability of SNR exceeding a chosen detection threshold $\rho_{\text {thr }}$ is proportional to $\exp \left(-\rho_{\mathrm{thr}}^{2} / 2\right)$. Because the many templates used in typical searches amount to repeated independent trials, $\rho_{\text {thr }}$ is routinely set to relatively large values (e.g., 5-10) to yield one false alarm per many years of data. The detectability of a signal $h$ is characterized by its optimal SNR $\rho(h ; h)$, which is the SNR that would be obtained on average over noise realizations for a perfectly matching template.

The noise $S_{X}(f)$ includes the confusion-noise foreground $S_{X}^{\text {conf }}(f)$ from unresolved sources (see Sec. 5), as well as instrument noise $S_{X}^{\text {inst }}$. For the unequal-arm Michelson observable $X$ in the (rather reasonable) limit of equal interferometer armlengths, $S_{X}^{\text {inst }}$ is given by (Vallisneri et al. 2008)

$$
S_{X}^{\text {inst }}=16 \sin ^{2}(2 \pi L f)\left[2\left(1+\cos ^{2}(2 \pi L f)\right) S_{\mathrm{pm}}^{\Delta f / f}(f)+S_{\mathrm{op}}^{\Delta f / f}(f)\right] .
$$

In these units $S_{\mathrm{pm}}^{\Delta f / f}=S_{\mathrm{pm}} /(2 \pi c f), S_{\mathrm{op}}^{\Delta f / f}(f)=S_{\mathrm{op}}(f) \times(2 \pi f / c)$.

To obtain the TDI response to a GW signal Eq. (5), we insert Eq. (5), multiplied by the appropriate phasing function and sky-position-dependent polarization tensors, into Eq. (B2). The response is modulated further by the heliocentric motion of the detector (which yields a time-dependent Doppler shift) and its yearly rotation in a plane inclined $60^{\circ}$ from the ecliptic (which generates sidebands at frequency multiples of $1 / \mathrm{yr}$ ) ${ }^{7}$ Both effects imprint sky-position and polarization angles into the TDI response; this structure makes it possible to resolve sufficiently strong GW sources that overlap in frequency space, and to determine their approximate sky position (see, e.g., Królak et al. 2004). The TDI response to a population of sources is given simply by the linear superposition of the responses to individual sources.

\section{FITS OF THE PARTIALLY SUBTRACTED CONFUSION-NOISE FOREGROUND}

Barack and Cutler (2004a, 2004b) codified the lore about the total noise of a LISA-like detector (i.e., the instrument noise plus Galactic-binary confusion noise) by providing a heuristic derivation for the well-known expression

$$
S_{h}^{\text {total,BC }}(f)=\min \left[S_{h}^{\text {inst }}(f) \times \mathrm{e}^{\kappa T^{-1} \mathrm{~d} N / \mathrm{d} f}, S_{h}^{\text {inst }}(f)+S_{h}^{\text {conf }, \mathrm{BC}}(f)\right],
$$

where they estimate

$$
\begin{gathered}
S_{h}^{\mathrm{conf}, \mathrm{BC}}(f)=1.4 \times 10^{-44}(f / \mathrm{Hz})^{-7 / 3} \mathrm{~Hz}^{-1}, \\
\frac{\mathrm{d} N}{\mathrm{~d} f}=2 \times 10^{-3}(f / \mathrm{Hz})^{-11 / 3} \mathrm{~Hz}^{-1},
\end{gathered}
$$

and $\kappa T^{-1} \simeq 1.5 / \mathrm{yr}$. As we discuss in App. A, under generic assumptions the unsubtracted Galactic-binary foreground will follow a $f^{-7 / 3}$ spectrum; this is confirmed in our simulations, with a coefficient $2.6 \times 10^{-44}$ for cases $1-3$ and 5 , and five times smaller for case 4 (see Fig. 3). After the subtraction of detectable sources, the total noise found in our simulations is fit better by a smaller amplitude, a slightly different exponent, and a smoother transition function:

$$
S_{h}^{\text {total }, \operatorname{sim}}(f)=S_{h}^{\text {inst }}+S_{h}^{\text {conf }, \operatorname{sim}} \times \tanh ^{\alpha}\left(\frac{1}{2} \beta \mathrm{d} N / \mathrm{d} f\right),
$$

\footnotetext{
7 Because "short LISA" orbits are very similar to those planned
} for classic LISA, this discussion is valid for both. Different orbits, 
with

$$
\begin{gathered}
S_{h}^{\text {conf }, \operatorname{sim}}(f)=1.4 \times 10^{-45}(f / \mathrm{Hz})^{-8 / 3} \mathrm{~Hz}^{-1}, \\
\frac{\mathrm{d} N}{\mathrm{~d} f}=5 \times 10^{-3}(f / \mathrm{Hz})^{-11 / 3} \mathrm{~Hz}^{-1} .
\end{gathered}
$$

For cases 1-3 and 5, and for our reference subtraction run (one year, one interferometric observable, $\rho_{\text {thr }}=5$ ), taking $\alpha=1, \beta=1 / \mathrm{yr}$ yields a good fit for a $5 \mathrm{Mkm}$ LISA-like detector, while $\alpha=0.7, \beta=1.2 / \mathrm{yr}$ is appropriate for $1 \mathrm{Mkm}$ arms. For the entire range of $\rho_{\text {eff }}$ that we probed, good fits are obtained by setting

$$
\begin{array}{llll}
\alpha=2-1.2\left(\rho_{\text {eff }} / 5\right)+0.2\left(\rho_{\text {eff }} / 5\right)^{2}, & \beta=\left[-0.2+1.5\left(\rho_{\text {eff }} / 5\right)-0.3\left(\rho_{\text {eff }} / 5\right)^{2}\right] / \mathrm{yr} & (5 \mathrm{Mkm}), \\
\alpha=1.6-1.2\left(\rho_{\text {eff }} / 5\right)+0.3\left(\rho_{\text {eff }} / 5\right)^{2}, & \beta=\left[0.3+1.3\left(\rho_{\text {eff }} / 5\right)-0.4\left(\rho_{\text {eff }} / 5\right)^{2}\right] / \mathrm{yr} & (1 \mathrm{Mkm}) .
\end{array}
$$

For case 4 , an acceptable fit follows from reducing $S_{h}^{\text {conf,sim }}$ by a factor of five. by

The equivalent confusion noise in the TDI $X$ combination (expressed as fractional-frequency fluctuations) is given

$$
S_{\mathrm{X}}^{\text {total }, \operatorname{sim}}(f)=\frac{3}{20} \frac{16 x^{2} \sin ^{2} x}{1+0.6 x^{2}} S_{h}^{\text {total }, \operatorname{sim}}(f), \text { with } x=2 \pi f L / c
$$

Here $3 / 20$ is the all-sky averaging $\left\langle F^{2}\right\rangle_{\hat{\mathbf{r}}, t}$ discussed above; $1+0.6 x^{2}$ is an approximation to the frequency-dependent interferometer response function; and $16 x^{2} \sin ^{2} x$ is the TDI $X$ transfer function. [In Barack and Cutler's convention (2004b), which we follow here, the average response function is included in the definition of the equivalent strain noise $S_{h}^{\text {total }}$, so that the sky-averaged SNR can be computed directly as $4 \operatorname{Re} \int|\tilde{h}|^{2} / S_{h}^{\text {total }}(f)$ d $f$; therefore $S_{h}^{\text {conf }}$, rather than $S_{h_{I}}^{\text {conf }}$, appears in $S_{h}^{\text {total }}$. By contrast, TDI observables already include the position-dependent instrument response, so it is appropriate to define the TDI noise $S_{\mathrm{X}}^{\text {total }}$ without the sky averaging.]

Due to the evolving orientation of the LISA-like formation with respect to the Galaxy, the Galactic foreground (before and after subtraction) has a strong seasonal variation in amplitude, almost constant across frequencies (Giampieri \& Polnarev 1997; Seto 2004, Edlund et al. 2005). For a single TDI $X$ combination, for both our detector configurations, for all scenarios, and for our assumptions about the relative orientation of detector and Galaxy at $t=0$ consistent with the standard MLDC LISA orbits (Arnaud et al.|2006)], this variation is fit well by

$$
S_{h}^{\mathrm{conf}, \operatorname{sim}}(f, t)=S_{h}^{\mathrm{conf}, \operatorname{sim}}(f) \times[1-0.21 \cos (2 \pi t / \mathrm{yr})-0.45 \cos (4 \pi t / \mathrm{yr})-0.05 \sin (2 \pi t / \mathrm{yr})+0.01 \sin (4 \pi t / \mathrm{yr})],
$$

where $S_{h}^{\text {conf,sim }}(f, t)$ denotes the power spectral density computed for data segments shorter than one year, but longer than the typical measurement timescale of a few hours. An equation analogous to Eq. (C9), with the same coefficients, holds also for the unsubtracted background $S_{h}(f, t)$.

\section{REFERENCES}

Amaro-Seoane, P., et al. 2012, eLISA: astrophysics and cosmology in the millihertz regime, arXiv:1201.3621

Arnaud, K. A., et al. 2006, in AIP Conference Proceedings, Vol. 873,625

Badenes, C., Mullally, F., Thompson, S. E., \& Lupton, R. H. 2009, ApJ, 707, 971

Barack, L., \& Cutler, C. 2004a, Phys. Rev. D, 69, 082005

—. 2004b, Phys. Rev. D, 70, 122002

Belczynski, K., Kalogera, V., \& Bulik, T. 2002, ApJ, 572, 407

Belczynski, K., Kalogera, V., Rasio, F., Taam, R., Zezas, A., Bulik, T., Maccarone, T., \& Ivanova, N. 2008, ApJ Supp. S., 174,223

Benacquista, M., DeGoes, J., \& Lunder, D. 2004, Class. Quantum Grav., 21, S509

Bender, P., \& Hils, D. 1997, Class. Quantum Grav., 14, 1439

Bildsten, L., Shen, K. J., Weinberg, N. N., \& Nelemans, G. 2007, ApJ, 662, L95

Boissier, S., \& Prantzos, N. 1999, MNRAS, 307, 857

Bovy, J., Rix, H.-W., \& Hogg, D. W. 2011, arXiv:1002.4677

Brown, W. R., Kilic, M., Allende Prieto, C., \& Kenyon, S. J. 2010, ApJ, 723, 1072

-. 2011, MNRAS, 411, L31

-. 2012, ApJ, 744, 142

Cornish, N. J., \& Littenberg, T. B. 2007, Phys. Rev. D, 76, 083006

Crowder, J., \& Cornish, N. J. 2004, Phys. Rev. D, 70, 082004

Cutler, C., \& Flanagan, É. E. 1994, Phys. Rev. D, 49, 2658

Edlund, J., Tinto, M., Królak, A., \& Nelemans, G. 2005, Phys. Rev. D, 71, 122003
Ergma, E. V., \& Fedorova, A. V. 1990, Ap\&SS, 163, 143 Estabrook, F. B., \& Wahlquist, H. D. 1975, Gen. Rel. Grav., 6, 439

Evans, C. R., Iben, Jr., I., \& Smarr, L. 1987, ApJ, 323, 129

Farmer, A., \& Phinney, E. 2003, MNRAS, 346, 1197

Farmer, A., \& Roelofs, G. 2010, arXiv:1006.4112

Fontaine, G., et al. 2011, ApJ, 726, 92

Giampieri, G., \& Polnarev, A. 1997, MNRAS, 291, 149

Han, Z. 1998, MNRAS, 296, 1019

Hils, D. 1998, in AIP Conference Proceedings, Vol. 456, 68

Hils, D., Bender, P., \& Webbink, R. 1990, ApJ, 360, 75

Hils, D., \& Bender, P. L. 2000, ApJ, 537, 334

Holberg, J. B., Sion, E. M., Oswalt, T., McCook, G. P., Foran, S., \& Subasavage, J. P. 2008, AJ, 135, 1225

Hughes, S. 2002, MNRAS, 331, 805

Hurley, J., Pols, O., \& Tout, C. 2000, MNRAS, 315, 543

Hurley, J., Tout, C., \& Pols, O. 2002, MNRAS, 329, 897

Iben, Jr., I., \& Tutukov, A. V. 1984, ApJS, 54, 335

Jennrich, O. 2009, Class. Quantum Grav., 26, 153001

Jurić, M., et al. 2008, ApJ, 673, 864

Kilic, M., Brown, W. R., Allende Prieto, C., Agüeros, M. A., Heinke, C., \& Kenyon, S. J. 2011a, ApJ, 727, 3

Kilic, M., Brown, W. R., Hermes, J. J., Allende Prieto, C., Kenyon, S. J., Winget, D. E., \& Winget, K. I. 2011b, arXiv:1109.6339

Królak, A., Tinto, M., \& Vallisneri, M. 2004, Phys. Rev. D, 70, 022003

Kroupa, P., Tout, C. A., \& Gilmore, G. 1993, MNRAS, 262, 545

Kulkarni, S. R., \& van Kerkwijk, M. H. 2010, ApJ, 719, 1123 
Levitan, D., et al. 2011, ApJ, 739, 68

Lipunov, V., Nazin, S., Panchenko, I., Postnov, K., \& Prokhorov, M. 1995, A\&A, 298, 677

Lipunov, V., \& Postnov, K. 1987, Astronomicheskii Zhurnal, 64, 438

Lipunov, V., Postnov, K., \& Prokhorov, M. 1987, A\&A, 176, L1 -. 1996, A\&A, 310, 489

Liu, J. 2009, MNRAS, 400, 1850

Liu, J., Zhang, Y., Han, Z., \& Zhang, F. 2010, Astrophysics and Space Science, 329, 297

Maggiore, M. 2007, Gravitational Waves. Vol. 1: Theory and Experiments (Oxford, UK: Oxford University Press)

Marsh, T. R. 2011, Class. Quantum Grav., 28, 094019

Marsh, T. R., Gaensicke, B. T., Steeghs, D., Southworth, J., Koester, D., Harris, V., \& Merry, L. 2010, arXiv:1002.4677

Marsh, T. R., Nelemans, G., \& Steeghs, D. 2004, MNRAS, 350, 113

Marsh, T. R., \& Steeghs, D. 2002, MNRAS, 331, L7

Maxted, P. F. L., \& Marsh, T. R. 1999, MNRAS, 307, 122

Mullally, F., Badenes, C., Thompson, S. E., \& Lupton, R. 2009, ApJ, 707, L51

Napiwotzki, R. 2009, Journal of Physics Conference Series, 172, 012004

Nelemans, G. 2009, Classical and Quantum Gravity, 26, 094030

Nelemans, G. 2012, LISA wiki (verification binaries): astro.ru.nl/ nelemans/dokuwiki/doku.php?id= verification_binaries:intro

Nelemans, G., Portegies Zwart, S. F., Verbunt, F., \& Yungelson, L. R. 2001a, AAP, 368, 939

Nelemans, G., Yungelson, L. R., \& Portegies Zwart, S. F. 2001b, AAP, 375,890

-. 2004, MNRAS, 349, 181

Nelemans, G., Yungelson, L. R., Portegies Zwart, S. F., \& Verbunt, F. 2001c, AAP, 365, 491

Peters, P., \& Mathews, J. 1963, Phys. Rev., 131, 435

Piro, A. L. 2011, ApJ, 740, L53

Podsiadlowski, P., Han, Z., \& Rappaport, S. 2001, astro-ph/0109171

Portegies Zwart, S., \& Yungelson, L. 1998, A\&A, 332, 173
Portegies Zwart, S. F., \& Verbunt, F. 1996, AAP, 309, 179

Postnov, K., \& Prokhorov, M. 1998, ApJ, 494, 674

Prince, T. A., et al. 2009, LISA: Probing the Universe with

Gravitational Waves, list.caltech.edu/mission_documents

Ramsay, G., Hakala, P., \& Cropper, M. 2002, MNRAS, 332, L7

Roelofs, G. H. A., Groot, P. J., Benedict, G. F., McArthur, B. E., Steeghs, D., Morales-Rueda, L., Marsh, T. R., \& Nelemans, G. 2007a, ApJ, 666, 1174

Roelofs, G. H. A., Nelemans, G., \& Groot, P. J. 2007b, MNRAS, 382,685

Ruiter, A. J., Belczynski, K., Benacquista, M., \&

Holley-Bockelmann, K. 2009, ApJ, 693, 383

Ruiter, A. J., Belczynski, K., Benacquista, M., Larson, S. L., \& Williams, G. 2010, ApJ, 717, 1006

Scannapieco, C., White, S. D. M., Springel, V., \& Tissera, P. B. 2011, MNRAS, 417, 154

Seto, N. 2002, MNRAS, 333, 469

Seto, N. 2004, Phys. Rev. D, 69, 123005

Shen, K. J., Kasen, D., Weinberg, N. N., Bildsten, L., \&

Scannapieco, E. 2010, ApJ, 715, 767

Siebert, A., et al. 2011, AJ, 141, 187

Solheim, J.-E. 2010, PASP, 122, 1133

Timpano, S. E., Rubbo, L. J., \& Cornish, N. J. 2006, Phys. Rev. $\mathrm{D}, 73,122001$

Toonen, S., Nelemans, G., \& Portegies Zwart, S. 2011, arXiv: 1101.2787

Tutukov, A., \& Yungelson, L. 1996, MNRAS, 280, 1035

Tutukov, A. V., \& Fedorova, A. V. 1989, Soviet Ast., 33, 606

Vallisneri, M. 2005, Phys. Rev. D, 72, 042003

Vallisneri, M. 2011, package fastbinary, lisasolve.googlecode.com, rev 458

Vallisneri, M., Crowder, J., \& Tinto, M. 2008, Class. Quantum Grav., 25, 065005

Verbunt, F., \& Rappaport, S. 1988, ApJ, 332, 193

Webbink, R., \& Han, Z. 1998, in AIP Conference Proceedings, Vol. 456,61

Webbink, R. F. 1984, ApJ, 277, 355

Yu, S., \& Jeffery, C. S. 2010, AAP, 521, A85 